\title{
Myoglobin primary structure reveals multiple convergent transitions to semi- aquatic life in the world's smallest mammalian divers
}

Kai He, ${ }^{* 1,2,3}$ Triston G. Eastman, ${ }^{1}$ Hannah Czolacz, ${ }^{4}$ Shuhao Li, ${ }^{1}$ Akio Shinohara, ${ }^{5}$ Shin-ichiro

Kawada, ${ }^{6}$ Mark S. Springer, ${ }^{7}$ Michael Berenbrink, ${ }^{*}, \dagger, 4$ and Kevin L. Campbell ${ }^{*, \dagger, 1}$

${ }^{1}$ Department of Biological Sciences, University of Manitoba, Winnipeg, MB, Canada

${ }^{2}$ School of Basic Medical Sciences, Southern Medical University, Guangzhou, Guangdong, China

${ }^{3}$ State Key Laboratory of Genetic Resources and Evolution, Kunming Institute of Zoology, Chinese Academy of Sciences, Kunming, Yunnan, China

${ }^{4}$ Department of Evolution, Ecology and Behaviour, University of Liverpool, Liverpool, United Kingdom

${ }^{5}$ Department of Bio-resources, Division of Biotechnology, Frontier Science Research Center, University of Miyazaki, Miyazaki, Japan

${ }^{6}$ Department of Zoology, Division of Vertebrates, National Museum of Nature and Science, Tokyo, Japan

${ }^{7}$ Department of Evolution, Ecology, and Organismal Biology, University of California, Riverside, CA, USA

*Corresponding authors: Email: hekai2018@smu.edu.cn, Michael.Berenbrink@liverpool.ac.uk, or Kevin.Campbell@umanitoba.ca

${ }^{\dagger}$ These authors contributed equally to this work 


\section{Abstract}

2 Identifying the phylogenomic underpinnings of specialized phenotypes that fueled transitions

3 into new adaptive zones is central to evolutionary biology but is often confounded by a

4 fragmentary fossil record, morphological convergence, and unresolved phylogenetic

5 relationships. The speciose mammalian order Eulipotyphla (e.g., moles, shrews, hedgehogs, solenodons) combines an unusual diversity of semi-aquatic, semi-fossorial, and fossorial forms

7 that arose from terrestrial forbearers, yet the ecomorphological pathways leading to these

8 lifestyles have been disputed for a century and more, calling for novel approaches. Here we resolve previously intractable eulipotyphlan intra-family relationships and establish the net surface charge of the oxygen-storing muscle protein myoglobin-readily determined from its primary structure-as a molecular signature to trace ancient lifestyle transitions based on protein sequence alone. Our analyses confidently resolve fossorial habits having evolved twice in talpid moles and reveal five independent origins of a semi-aquatic lifestyle in the order housing the world's smallest endothermic divers.

\section{Introduction}

A fundamental challenge of evolutionary biology is to understand the phylogenomic foundations of biochemical and physiological specializations that have enabled organisms to proliferate into new adaptive zones. While species of most mammalian orders generally occupy a single niche, members of a few mammalian lineages have radiated into a diverse range of environmental habitats. The order Eulipotyphla is one such assemblage, in that terrestrial forms repeatedly evolved into high-altitude, scansorial (climbing), fossorial (subterranean), semifossorial, and semi-aquatic niches (Burgin and He 2018; He, et al. 2017).

The fourth largest among the 27 mammalian orders after Rodentia, Chiroptera, and Cetartiodactyla, the eulipotyphlan clade consists of 527 recognized species of small insectivorous mammals distributed among four extant families (Burgin, et al. 2018): Erinaceidae

27 (hedgehogs, gymnures, and moonrats), Soricidae (shrews), Talpidae (moles, shrew moles, shrewlike moles, and desmans), and Solenodontidae (solenodons). A fifth family (Nesophontidae, Caribbean 'island-shrews') only became extinct in the past 500 years following the arrival of

30 Europeans in the West Indies (MacPhee, et al. 1999). Fossil evidence supports terrestrial habits as ancestral for Eulipotyphla, with shrew-like moles, erinaceids, and the majority of shrews 
retaining morphological characteristics (e.g., slender legs and a long, pointed mouse-like snout; fig. 1) effective for hunting and avoiding predation on land (Churchfield 1990; Nowak 1999).

Semi-aquatic moles and shrews possess a stream-lined body and limb adaptations for underwater locomotion (Nowak 1999; Burgin and He 2018), with desmans having an outward appearance more similar to that of semi-aquatic rodents (e.g., muskrat) than to that of any other eulipotyphlan mammal (fig. 1). By contrast, fossorial moles have evolved powerful forelimbs that are held out to the sides of their tube-shaped bodies and have large, spade-like hands facing posteriorly and oriented vertically allowing them to be effective burrowers (Gorman and Stone 1990). Finally, semi-fossorial shrews, solenodons, and shrew moles exhibit specializations inbetween that of terrestrial and fossorial lineages, often retaining pointed mouse-like snouts yet possessing forelimbs that are oriented downwards and backwards to facilitate digging (Gorman and Stone 1990; Nowak 1999).

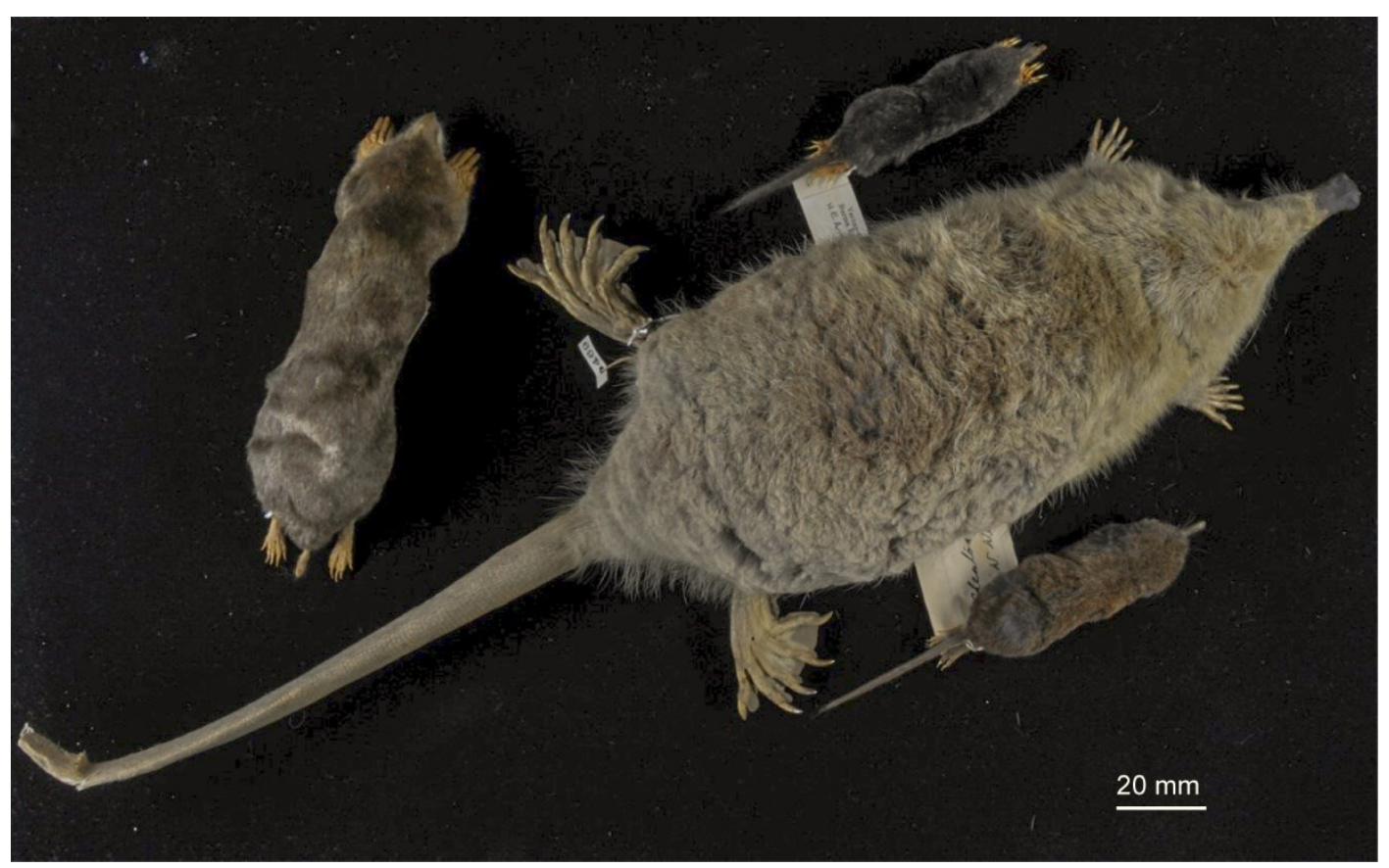

Fig. 1. Museum specimen photos illustrating the four major ecomorphotypes within the order Eulipotyphla. Representative terrestrial (shrew-like mole, Uropsilus soricipes; bottom right), semi-aquatic (Russian desman, Desmana moschata; right centre), strictly fossorial (Eastern mole, Scalopus aquaticus; left), and semi-fossorial (Chinese long-tailed mole, Scaptonyx fusicaudus; top centre) talpid mole species are given. Photo by Kai He. 
While the lifestyle of extant eulipotyphlan mammals is easily predicted based on external morphological features alone (e.g., Woodman and Gaffney 2014; Sansalone, et al. 2016; Sansalone, et al. 2019), definitive assignments of transitional evolutionary stages have remained elusive due to an incomplete, fragmentary fossil record (Hickman 1984; Sánchez-Villagra, et al. 2006; Piras, et al. 2012; Hooker 2016). Additionally, because morphological specializations coincide with lifestyle across the different clades of eulipotyphlan mammals, it has been difficult to discern whether fossorial and semi-aquatic specializations found in Eurasian and North American moles arose in a common ancestor or are due to convergent evolution (Schwermann and Thompson 2015; He, et al. 2017). As a result, long-standing questions persist regarding the evolutionary pathways leading to the diverse ecomorphological variation seen in eulipotyphlan mammals.

For example, examinations of shoulder anatomy (Campbell 1939) and skeletal musculature (Whidden 1999) indicated that the shoulder and humeroclavicular joint morphology of fossorial talpid species aligned more closely with semi-aquatic locomotion, suggesting that the ancestor of Talpinae moles was semi-aquatic, which we here term the 'aquatic mole' hypothesis. Conversely, Reed (1951) analyzed anatomical features from four talpid genera and proposed that semi-fossorial and fossorial forms evolved from a terrestrial ancestor and that the semi-aquatic lifestyle of desmans was secondary to a semi-fossorial phase, a view also shared by Grand et al. (1998). Skeletal and fossil evidence also suggested that a period of strictly fossorial life preceded the invasion of semi-aquatic habits by the ancestors of star-nosed moles (Condylura cristata) (Hickman 1984; Grand, et al. 1998; Sanchez-Villagra, et al. 2006; Piras, et al. 2012; Hooker 2016), though Sansalone et al. (2016) proposed that the semi-aquatic lifestyle of this genus instead represents an autapomorphy from a semi-fossorial (shrew mole-like) ancestor.

Adaptive morphology and behavioural/physiological specializations for diving have also been well documented for members of the family Soricidae (Hutterer 1985; Catania, et al. 2008; Burgin and He 2018), though uncertainties regarding the evolution of semi-aquatic habits in this clade are also pervasive. For example, semi-aquatic members of four genera, Sorex (North American water shrews), Neomys (European water shrews), Chimarrogale (Asian water shrews), and Nectogale (Elegant water shrews) show a graded series of progressively greater morphological adaptations for underwater foraging (Hutterer 1985), yet phylogenetic analyses strongly refute a common ancestry of aquatic exploitation (He et al. 2010). Ancestral-state 
reconstructions and the placement of Chimarrogale + Nectogale as sister-taxa instead provided support for semi-aquatic habits having evolved three times (He et al. 2010), as opposed to four convergent invasions (Churchfield 1990). However, based on associated fauna/flora and paleoecological indicators of one of the oldest fossil nectogaline genera (Asoriculus), it has been hypothesized that a shared Late Miocene ancestor of Neomys, Chimarrogale, and Nectogale may have been adapted to humid environments with permanent open water (Rofes and CuencaBescos 2006), thus allowing the possibility that semi-aquatic habits evolved only twice in shrews. Taken together, potential morphological convergence and an incomplete fossil record have precluded reliable conclusions to be made regarding the evolutionary pathways underlying the origins of the diverse phenotypes of eulipotyphlan mammals.

The monomeric oxygen-binding protein myoglobin, which plays an essential role in $\mathrm{O}_{2}$ storage and facilitates $\mathrm{O}_{2}$ diffusion within the heart and skeletal muscle of most vertebrates, provides an intriguing, objective molecular target to address this question. Indeed, the $\mathrm{O}_{2}$ storage function of myoglobin has long been known to contribute integrally to the mammalian dive response, with maximum active submergence times of birds and mammals being strongly correlated to muscle myoglobin concentrations (Noren and Williams 2000; Lestyk, et al. 2009; Ponganis, et al. 2011). Links between the molecular evolution of this molecule and aquatic life had already been suspected for a half century or more (Scholander 1962; Romero-Herrera, et al. 1973), leading to the speculation that "there might be functional reasons, perhaps associated with diving" underlying the presence of parallel amino acid replacements in the myoglobins of seals and cetaceans (Romero-Herrera, et al. 1978). Mirceta et al. (2013) extended this work to reveal that maximal muscle myoglobin concentration was mechanistically linked to myoglobin net surface charge $\left(Z_{\mathrm{Mb}}\right)$ in mammals via adaptive changes in primary structure, with convergent increases in $Z_{\mathrm{Mb}}$ found in members of all eight lineages with an extended aquatic/semi-aquatic evolutionary history. This trait presumably represents an adaptive response to combat the general propensity for proteins to precipitate at high concentration, thereby allowing for advantageous elevations of this muscle $\mathrm{O}_{2}$ store without deleterious self-aggregation (Mirceta, et al. 2013). An increase in $Z_{\mathrm{Mb}}$ may also reduce the tendency of the myoglobin peptide (apomyoglobin) to

112 formation of an aggregate to be energetically unfavourable (Samuel, et al. 2015; Isogai, et al. 
$Z_{\mathrm{Mb}}$ presumably represent an evolutionary trade off because there is less room for contractile units (sarcomeres) and organelles (e.g., mitochondria) in muscle tissue as more energy and space is allocated to myoglobin. Therefore, an increased $Z_{\mathrm{Mb}}$ only offers an advantage to species experiencing hypoxic episodes as for example occurs during breath-hold dives when access to environmental air is impeded. Importantly, the finding that $Z_{\mathrm{Mb}}$ and maximal myoglobin concentrations are only slightly elevated within mammals that live at high elevations or have fossorial lifestyles (Mirceta, et al. 2013) suggests that this locus may be useful to discern past semi-aquatic versus high-altitude/fossorial evolutionary histories within eulipotyphlan mammals.

Support for the above contention is provided by the molecular modelling and phylogenetic reconstruction of $Z_{\mathrm{Mb}}$ in six eulipotyphlan species (Mirceta, et al. 2013), which revealed convergent elevations in $Z_{\mathrm{Mb}}$ in both semi-aquatic taxa included in the dataset-the American water shrew (Sorex palustris) and the star-nosed mole. While myoglobin concentration data is sparse for eulipotyphlan mammals, available data indicates that myoglobin levels are also higher in these two species relative to non-aquatic forms (McIntyre, et al. 2002; Gusztak 2008).

Notably, $Z_{\mathrm{Mb}}$ was reconstructed to secondarily decline in the branch containing the single strictly fossorial talpid species (Mirceta, et al. 2013), thus providing tentative support for the 'aquatic mole' hypothesis. The sparse taxon sampling in the cited study (two moles, three shrews, and a hedgehog), however, did not account for the broad phylogenetic and ecomorphological diversity within these families. Additionally, current phylogenetic hypotheses for Eulipotyphla lack definitive resolution below the family level (He, et al. 2010; He, et al. 2017) thereby precluding reliable ancestral reconstructions. To overcome these shortcomings, we used a capture hybridization approach to target coding sequences of myoglobin together with 25 tree-of-life genes from 61 eulipotyphlan DNA libraries (44 moles, 11 shrews, 5 hedgehogs, and 1 solenodon) that included representatives from all seven recognized semi-aquatic genera within this order. We then tested whether $Z_{\mathrm{Mb}}$ is elevated within members of all living genera of semiaquatic Eulipotyphla, and if so, whether there is a significant correlation between this lifestyle and $Z_{\mathrm{Mb}}$. Having shown that these important conditions are met, we then traced $Z_{\mathrm{Mb}}$ across eulipotyphlan evolutionary history, thereby allowing us to determine when and how many times semi-aquatic specializations for increased dive durations evolved in both shrews and moles, and evaluate the merit of the 'aquatic mole' hypothesis versus alternative evolutionary scenarios of talpid lifestyle evolution. 


\section{Results}

146 Phylogenetic relationships within Eulipotyphla

147 To obtain mammalian tree-of-life genes (Meredith, et al. 2011) for phylogenetic estimation,

148 we conducted in-solution probe-hybridization for segments of 25 single-copy genes from 61

149 eulipotyphlan DNA libraries (supplementary table S1). Twenty-three of these loci were

150 efficiently captured, with usable sequence obtained from 51 to 61 libraries per locus, equivalent

151 to a $\sim 95 \%(1330 / 1403)$ success rate (supplementary table S2). By contrast, probes for two gene

152 segments only successfully hybridized to 21 and 15 of the 61 libraries, respectively, and were

153 subsequently not included in the phylogenetic analyses. Specifically, we failed to capture the

154 prepronociceptin (PNOC) gene for any shrew and many of the talpid species (supplementary

155 table S2). Probes for the interphotoreceptor retinoid binding protein (IRBP) also failed to retrieve

156 any sequence from the solenodon and 37 of the 38 non-uropsiline talpid specimens. Notably, the

157 putative $234 \mathrm{bp}$ IRBP fragment recovered from True's shrew mole (Dymecodon pilirostris)

158 contained gaps and premature stop codons, suggesting this gene was inactivated in the common

159 ancestor of the subfamily Talpinae. Similarly, the Chinese mole shrew (Anourosorex squamipes)

160 IRBP sequence contained premature stop codons and was presumed to be non-functional. After

161 incorporating orthologous tree-of-life sequence data from ten additional eulipotyphlan specimens

162 and five outgroup species downloaded from GenBank (see Methods for details), our 76 specimen

163 dataset (71 eulipotyphlans) resulted in a final alignment of 39,414 bp.

Fig. 2. Time calibrated Bayesian phylogenetic tree of Eulipotyphla based on a concatenated alignment of

16723 nuclear genes (outgroups not shown). Branch lengths represent median ages. Node bars indicate the

$16895 \%$ confidence interval [CI] for each clade age. Unless specified, all relationships are highly supported.

169 Relationships weakly supported in concatenation Bayesian and maximum likelihood ( $\mathrm{PP}<0.97$ and/or BS

$170<80$ : \#) as well as *BEAST and ASTRAL coalescent analyses (C-PP $<0.97$ and/or C-BS $<80: *)$ are

171 indicated. An alternative position was recovered for Soricini in the ASTRAL tree (supplementary fig. S2),

172 while both coalescent analyses (ASTRAL, *BEAST) favored Episoriculus monophyly (supplementary

173 figs. S2, S3). (next page) 
bioRxiv preprint doi: https://doi.org/10.1101/2021.01.14.426706; this version posted February 2, 2021. The copyright holder for this preprint (which was not certified by peer review) is the author/funder, who has granted bioRxiv a license to display the preprint in perpetuity. It is made available under aCC-BY-NC-ND 4.0 International license.

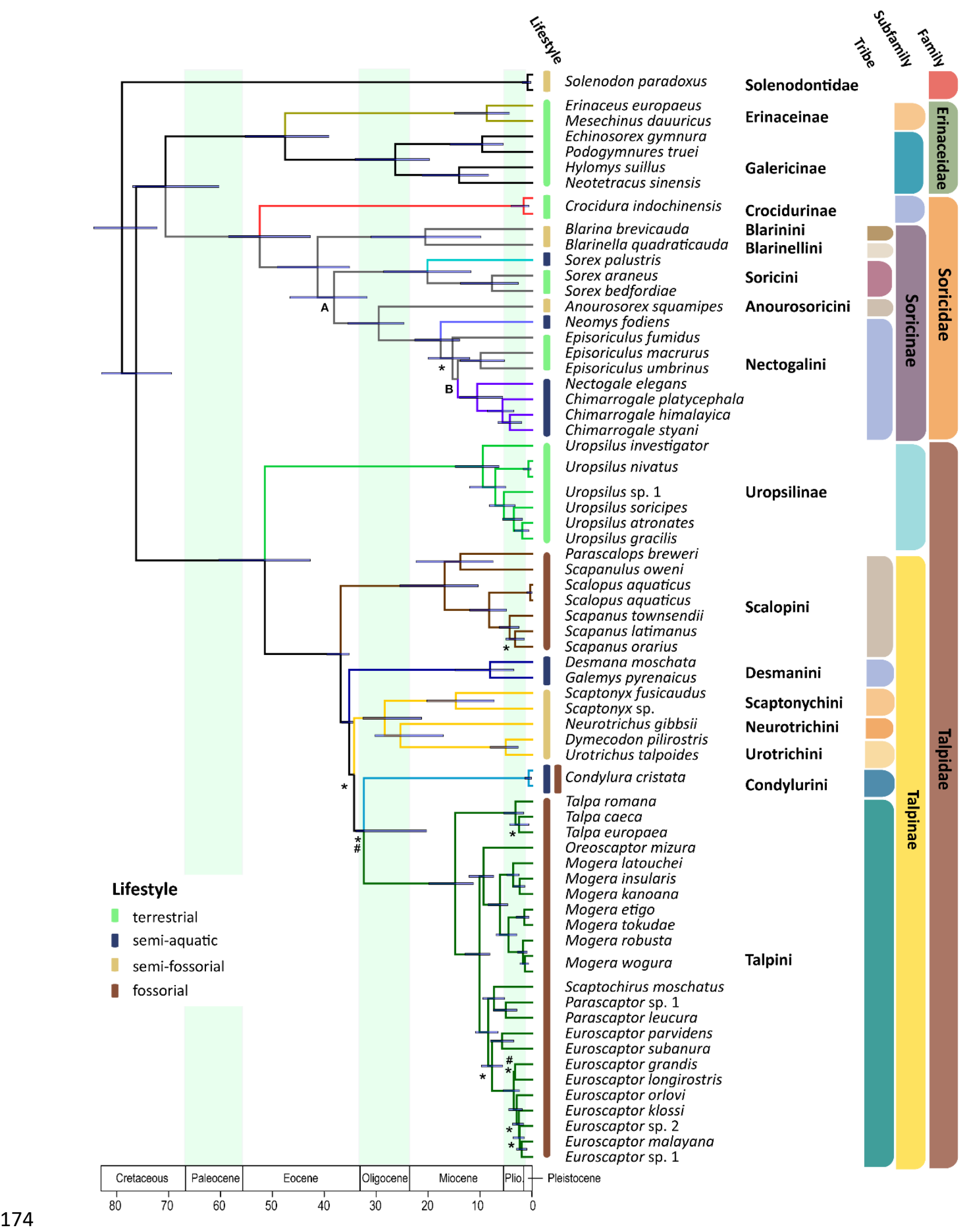


To estimate evolutionary relationships, we used maximum likelihood (RAxML) and Bayesian (BEAST) approaches on the concatenated alignment, and coalescent-based species tree methods (ASTRAL-III, *BEAST) on the 23 individual gene trees. These analyses resulted in highly congruent topologies (fig. 2 and supplementary figs. S1, S2, S3), with family level relationships corresponding to those obtained in recent eulipotyphlan (Brace, et al. 2016; Springer, et al. 2018) and mammal wide studies (Meredith, et al. 2011; Esselstyn, et al. 2017). Higher-level relationships within Soricidae and Erinaceidae also corresponded closely with previous studies on these families (He, et al. 2010; He, et al. 2012). Principal among these is the non-sister relationship of the Nectogalini water shrew clades Neomys and Chimarrogale + Nectogale, as the latter was strongly supported as sister to terrestrial Episoriculus by RAxML (bootstrap support $[\mathrm{ML}-\mathrm{BS}]=97$; supplementary fig. $\mathrm{S} 1$ ), BEAST (posterior probability $[\mathrm{PP}]=$ 1.0; fig. 2), ASTRAL-III (coalescent bootstrap support based on gene-wise resampling [C-BS] = 76; supplementary fig. S2), and *BEAST (posterior probability [C-PP] $=0.99$; supplementary fig. S3). By contrast, previously intractable relationships within Talpidae are now resolved, with the interrelationships among fossorial and semi-aquatic clades consistently recovered (fig. 2 and supplementary figs. S1, S2, S3). Specifically, desmans are placed sister to a clade containing shrew moles, star-nosed moles, and Eurasian fossorial moles $(\mathrm{BS}=100, \mathrm{PP}=1.0, \mathrm{C}-\mathrm{BS}=87, \mathrm{C}$ $\mathrm{PP}=0.97)$, with Condylurini and Talpini recovered as sister lineages $(\mathrm{BS}=72, \mathrm{PP}=1.0, \mathrm{C}-\mathrm{BS}$ $=52, \mathrm{C}-\mathrm{PP}=0.93)$. North American fossorial Scalopini moles are also supported as sister to all other Talpinae moles with high support scores $(\mathrm{BS}=97, \mathrm{PP}=1.0, \mathrm{C}-\mathrm{BS}=87, \mathrm{C}-\mathrm{PP}=0.97)$. Accordingly, a sister group relationship of fully fossorial Scalopini and Talpini moles was statistically rejected by the Shimodaira-Hasegawa (SH) test $(p<0.01$, supplementary table S3). The only minor incongruences among phylogenies was the position of the tribe Soricini and of the Taiwanese brown-toothed shrew (Episoriculus fumidus) within Nectogalini in the ASTRAL tree (fig. 2 and supplementary figs. S1, S2, S3).

\section{Myoglobin primary structure}

Complete myoglobin coding sequences (465 base pairs including initiation and stop codons) were obtained for 55 eulipotyphlan species ( 38 moles, 14 shrews, 2 erinacids, and 1 solenodon) using capture-hybridization, transcriptome sequencing, genome mining, and PCR approaches. Conceptual translations revealed the expected 153 amino-acid peptides in most cases. However, 
the translated myoglobin proteins of the Chinese mole shrew, both members of the genus Scaptonyx, and the two desman genera are only 152 amino acids in length. In every case the shorter myoglobin sequences are the result of $3 \mathrm{bp}$ deletions in exon 3 that corresponded to residue position 121 (supplementary table S4) and shorten the loop between helices $\mathrm{G}$ and $\mathrm{H}$ from 6 to 5 residues. These deletions were confirmed for each lineage using at least two of the three sequencing approaches noted above. To our knowledge, a similar deletion was previously only known for three bird of prey species (Enoki et al. 2008), but we have detected it also in the predicted myoglobin sequences of the draft genomes of several burrowing species of the order Rodentia (data not shown), notably including the fully fossorial Transcaucasian and Northern mole voles (Ellobius lutescens and E. talpinus, respectively; Mulugeta, et al. 2016).

\section{Myoglobin homology modelling}

Homology modelling of myoglobin structure was conducted for one extant species from each of the five diving lineages together with that predicted for the last common eulipotyphlan ancestor based on ancestral sequence reconstruction (see next section). This analysis confirmed that all charge-changing substitutions were located at the solvent-exposed surface of the protein (supplementary table S4). These comparisons further suggested that deletion of position 121 in the loop between helices $\mathrm{G}$ and $\mathrm{H}$ in the myoglobin of the Russian desman has minimal effect on the tertiary structure of the protein (supplementary fig. S5). In addition to their effect on $Z_{\mathrm{Mb}}$, some of the charge-increasing substitutions were associated with a complex re-arrangement of the network of salt bridges in the tertiary structure of the protein. Thus, for example, during the evolution of the Russian desman, arguably the most aquatic of extant Eulipotyphla, the two neighbouring substitutions Gln ${ }^{26} \rightarrow \operatorname{Arg}^{26}$ (charge-increasing) and Glu $^{27} \rightarrow \operatorname{Asp}^{27}$ (charge-neutral) in the B-helix allow for the formation of a new intra-helical salt bridge between $\operatorname{Arg}^{26}$ and $\mathrm{Asp}^{27}$, while at the same time breaking the inter-helical salt bridge between $\mathrm{Glu}^{27}$ and $\mathrm{Lys}^{118}$ in the Band G-helices, respectively (fig. 3). However, charge-decreasing $\mathrm{Asn}^{35} \rightarrow \mathrm{Asp}^{35}$ followed by charge-increasing $\mathrm{Gln}^{113} \rightarrow$ Lys $^{113}$ subsequently again tethered the B- and G-helices to each other by the salt bridge $\mathrm{Asp}^{35}$ - Lys ${ }^{113}$. Further, the removal of a negative charge by the substitution $\mathrm{Asp}^{44} \rightarrow \mathrm{Ala}^{44}$ destroyed a salt bridge between $\mathrm{Asp}^{44}$ and Lys ${ }^{47}$ in the CD-corner of the protein, thereby potentially affecting the flexibility of the loop between the C-and D-helices (fig. 3). However, a detailed assessment of any changes in the folding stability of the proteins that are 
associated with the identified charge-changing substitutions, let alone with any charge neutral replacements (see, e.g., Isogai, et al. 2018), is difficult and beyond the scope of this study, not least because of the potentially opposing effects of salt bridge formation and the associated desolvation of charges for the folding stability of proteins (for discussion see Bosshard, et al. 2004).

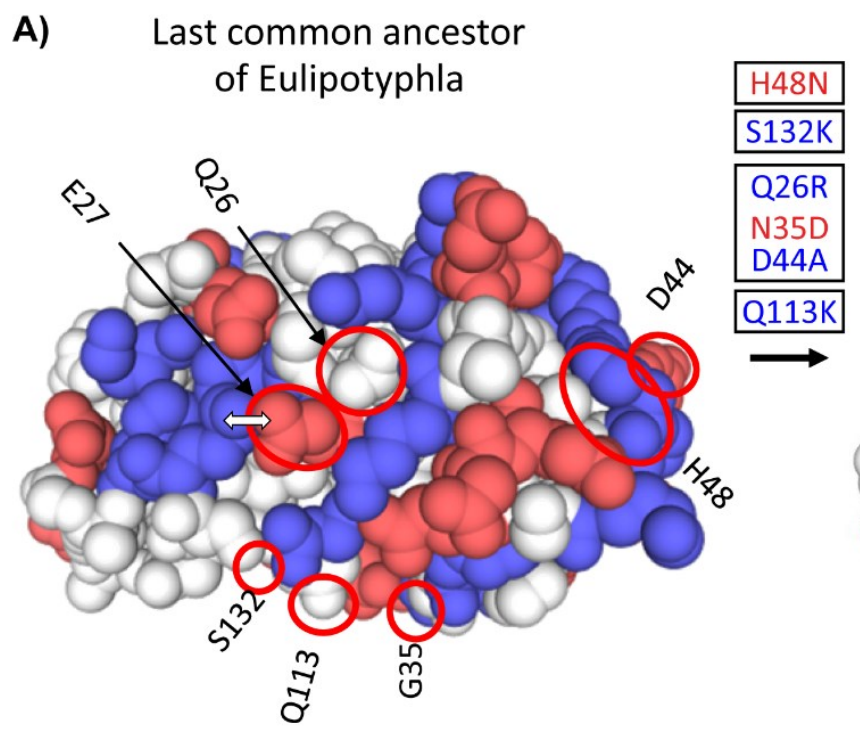

\section{B) Desmana moschata}

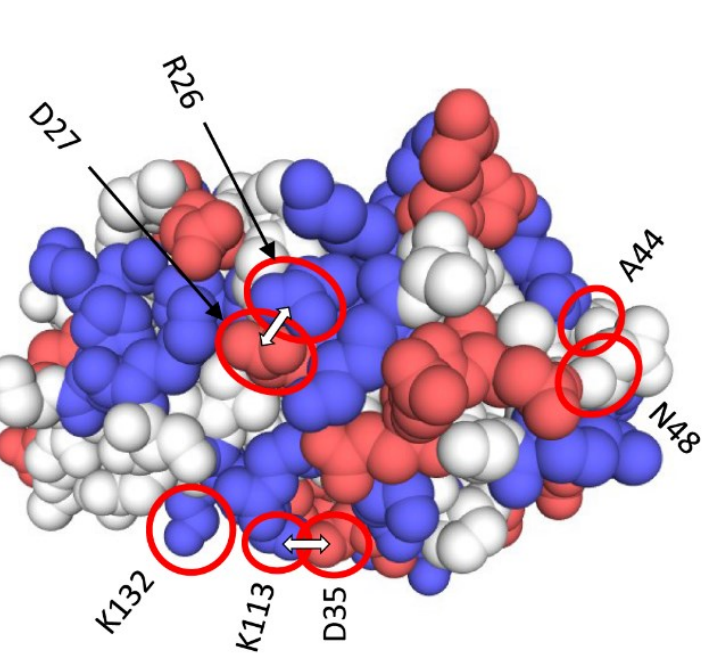

Fig. 3. Three-dimensional structural models of myoglobin in (A) the last common ancestor of Eulipotyphla and (B) the semi-aquatic Russian desman (Desmana moschata) obtained by homology modelling using the SWISS-MODEL server (Waterhouse, et al. 2018). The structure of the last common ancestor of the group was modelled based on results of an amino acid sequence reconstruction (see text for details). Ancestral (left) and derived (right) states of chargechanging amino acid replacements are circled and indicated with positional number and oneletter amino acid code. Blue and red color indicate amino acids with positively $(\mathrm{H}$, His; $\mathrm{K}$, Lys; R, Arg) and negatively charged amino acid side chains (D, Asp; E, Glu), respectively. White double arrows indicate surface amino acid side chains involved in salt bridges that are affected by charge-changing substitutions. Text boxes indicate the reconstructed temporal order (top to bottom) of charge decreasing and charge increasing amino acid substitutions (red and blue font, respectively) in the Desmana lineage in one letter code from ancestral (left) to derived (right) separated by positional number. Note that charge neutral substitutions (e.g., G35N), are not given in the text boxes. 


\section{Electrophoretic mobility and ancestral reconstruction of $Z_{M b}$}

Without exception, the modelled $Z_{\mathrm{Mb}}$ values of extant semi-aquatic taxa (2.07 to 3.07) were substantially higher than those of terrestrial Eulipotyphla (-0.46 to 0.63$)$, with fossorial species generally exhibiting intermediate $Z_{\mathrm{Mb}}$ values (typically 1.07; fig. 4A and supplementary fig. S6). To assess the reliability of our $Z_{\mathrm{Mb}}$ determinations, we measured the electrophoretic mobility of the myoglobin band of muscle extracts from two semi-aquatic, two strictly fossorial, and one terrestrial eulipotyphlan species (fig. 4B). The close correspondence between the two variables validates $Z_{\mathrm{Mb}}$ as a molecular marker for inferring present and past semi-aquatic habits in Eulipotyphla.

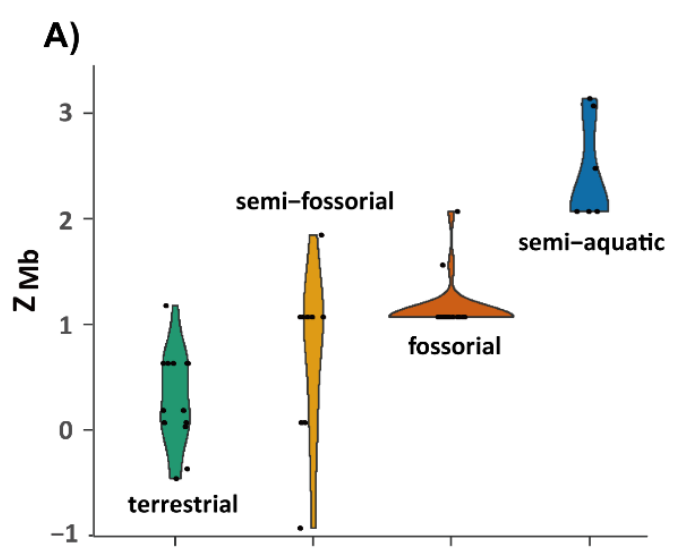

Lifestyles

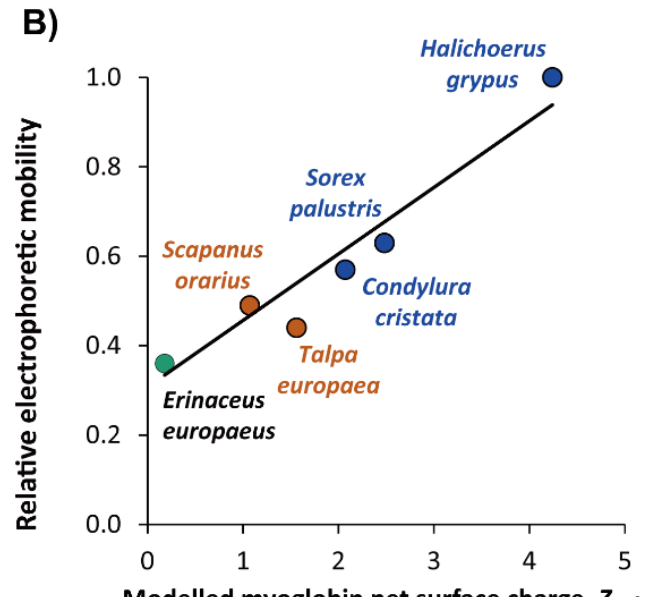

Modelled myoglobin net surface charge, $Z_{\mathrm{Mb}}$

Fig. 4. (A) Violin plot showing the distribution (y-axis) and probability density (x-axis) of modelled myoglobin net surface charge, $Z_{\mathrm{Mb}}$, among living species (black dots) of the four prevalent eulipotyphlan ecomorphotypes. (B) Correlation between $Z_{\mathrm{Mb}}$ and electrophoretic mobility of native myoglobin from five eulipotyphlan insectivores; data from the grey seal (Halichoerus grypus) is added for comparison. $Z_{\mathrm{Mb}}$ was calculated as the sum of the charge of all ionisable groups at $\mathrm{pH} 6.5$ by modelling myoglobin primary structures onto the tertiary structure and using published, conserved, site-specific ionization constants (McLellan 1984; Mirceta, et al. 2013). Electrophoretic mobility was assessed relative to the mobility of grey seal myoglobin using native polyacrylamide gel electrophoresis of heart or skeletal muscle protein extracts of the indicated species. Green, orange, brown, and blue areas (A) or symbols and fonts (B) indicate terrestrial, semi-fossorial, fossorial, and semi-aquatic/aquatic species, respectively. Phylogenetic Generalized Least Squares analysis in panel (B) revealed a highly significant positive correlation $\left(R^{2}=0.897, p<0.005\right)$ between the two parameters (solid line, $\left.y=0.1488 x+0.3075\right)$. 
Our myoglobin nucleotide and amino acid gene trees retrieved few well-supported evolutionary history that deviated from the species trees (supplementary figs. S8A, B), which would potentially result in erroneous ancestral reconstructions (Hahn and Nakhleh, 2016). We thus conducted a maximum likelihood ancestral amino acid sequence reconstruction using the species tree in fig. 1 as the phylogenetic backbone and the best fitting model of the Dayhoff amino acid substitution matrix with a gamma distribution of rate variation among sites. This analysis yielded highly supported ancestral amino acid identities across all 153 residues and 58 internal nodes of the species tree (supplementary fig. S7A), which was presumably due to our dense taxon sampling and the relatively highly conserved primary structure of mammalian myoglobins. Of the $8,874(=58 \times 153)$ reconstructed ancestral sites on the species tree, 8,799 $(99.15 \%)$ had maximal probabilities of reconstructed amino acid identities of $p>0.95$ under the given phylogeny and amino acid substitution model. In 50 cases $(0.55 \%)$, alternatively reconstructed amino acids with $p>0.05$ were of the same charge and thus did not affect the calculated $Z_{\mathrm{Mb}}$ values. Only in 25 cases $(0.28 \%)$, one or more alternatively reconstructed amino

297 acids with $p>0.05$ carried a different charge from the most probable amino acid at that site.

298 However, even in those cases this usually had only a minimal effect on $Z_{\mathrm{Mb}}( \pm 0.11 ; 3$ sites $)$, or the summed probabilities of alternative charge states were comparatively small $(p \leq 0.21 ; 23$ sites) such that we regard both the results of the ancestral sequence reconstruction and of the resulting values of $Z_{\mathrm{Mb}}$ as robust. This is supported by generally congruent results obtained by codon-based ancestral sequence reconstructions (supplementary fig. S7B), which primarily deviated from the above empirical amino acid matrix-based method at some deeper nodes, where

304 a higher number of negatively charged amino acid residues (and thus anomalously low values for $Z_{\mathrm{Mb}}$; e.g., -1.93) were reconstructed relative to those obtained for extant mammalian species 306 (Mirceta, et al. 2013; this study). Consequently, the following discussion largely focuses on the $307 Z_{\mathrm{Mb}}$ reconstruction using the empirical Dayhoff substitution matrix (supplementary fig. S7A), 308 which moreover - in contrast to the codon-based analysis (supplementary fig. S7B) —includes 309 the effects of purifying selection acting on replacements with dissimilar amino acid properties. 


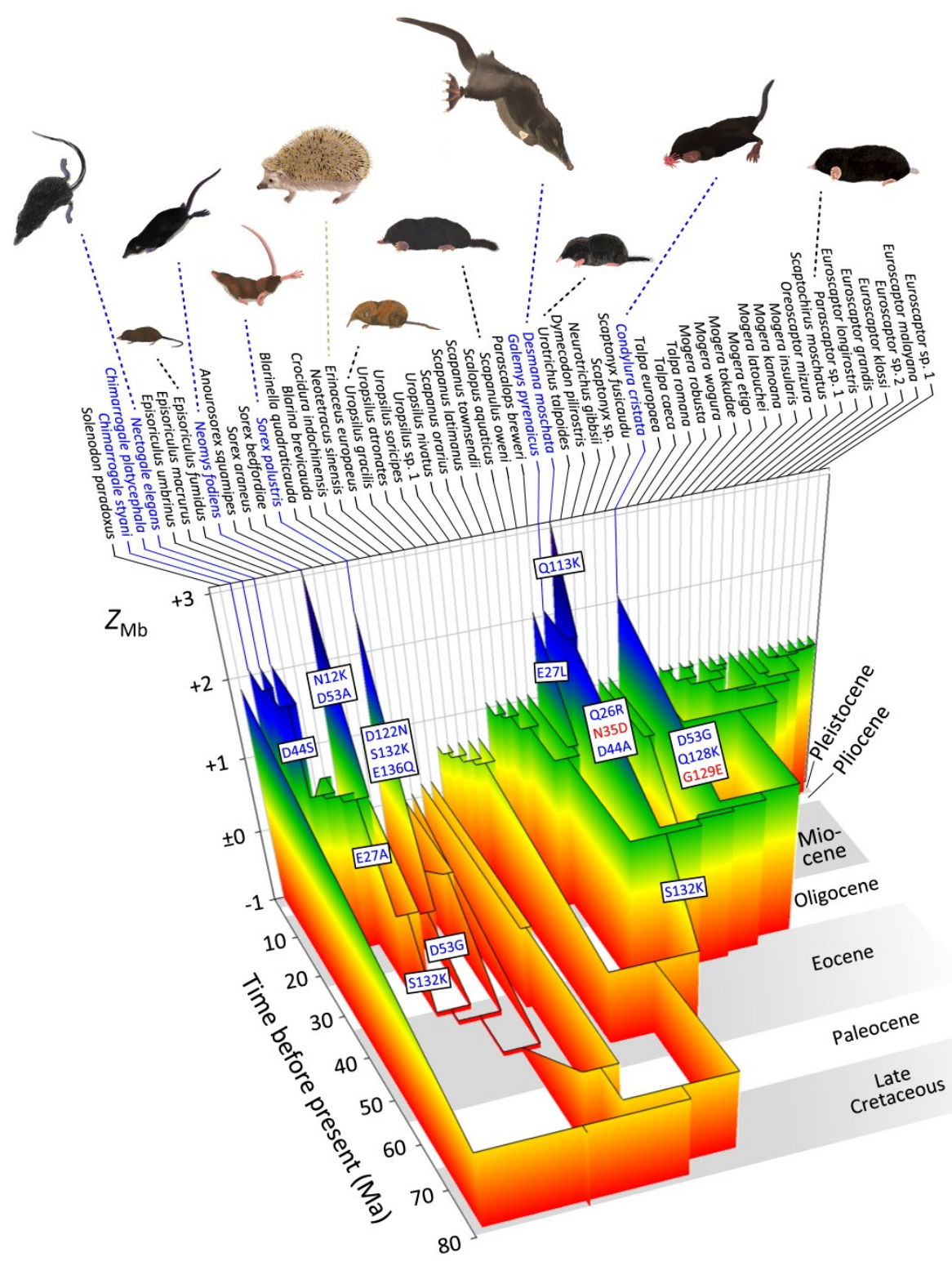

311 Fig. 5. Evolutionary reconstruction of myoglobin net surface charge $Z_{\mathrm{Mb}}$ in 55 eulipotyphlan

312 insectivores mapped onto the time calibrated phylogeny of Fig. 2. Ancestral $Z_{\mathrm{Mb}}$ was modelled

313 from primary structures as in Fig. 3 and after maximum likelihood ancestral sequence

314 reconstruction. Major charge increasing (blue font) and charge decreasing (red font) amino acid

315 substitutions, from ancestral to derived and separated by positional number, inferred for the

316 immediate ancestry of semi-aquatic species (blue font) are indicated in textboxes alongside the

317 respective branches. Grey and white background shading indicates geologic epochs. See

318 supplementary Fig. S7A for a complete account of charge-changing substitutions, reconstructed

$319 Z_{\mathrm{Mb}}$ values, and outgroup information. Paintings of representative species by Umi Matsushita. 
Ancestral $Z_{\mathrm{Mb}}$ estimates arising from the best fitting amino-acid based reconstruction model indicated that the most recent common ancestor of Eulipotyphla displayed a $Z_{\mathrm{Mb}}$ of 0.18 . Within Soricidae, distinct increases in $Z_{\mathrm{Mb}}$ were found on the branches leading to the three semi-aquatic clades (fig. 5). Specifically, the $Z_{\mathrm{Mb}}$ of the American water shrew (Sorex palustris) branch increased from -0.46 to 2.48 and was characterized by three integral $(+1)$ charge increasing residue replacements $\left(\mathrm{Asp}^{122} \rightarrow \mathrm{Asn}^{122}, \mathrm{Ser}^{132} \rightarrow \mathrm{Lys}^{132}\right.$, and Glu ${ }^{136} \rightarrow \mathrm{Gln}^{136}$; supplementary fig. S4A). The other three genera of water shrews reside in the tribe Nectogalini, the stem branch of which evolved a single charge increasing substitution $\left(\mathrm{Glu}^{27} \rightarrow \mathrm{Ala}^{27}\right)$ and thus had a $Z_{\mathrm{Mb}}$ of 1.07 (fig. 5). The European water shrew branch (Neomys fodiens) subsequently acquired two charge increasing substitutions ( $\mathrm{Asn}^{12} \rightarrow \mathrm{Lys}^{12}, \mathrm{Asp}^{53} \rightarrow \mathrm{Ala}^{53} ; Z_{\mathrm{Mb}}=3.07$; supplementary fig. S4B), while the common ancestor of Nectogale + Chimarrogale evolved a separate charge increasing replacement $\left(\mathrm{Asp}^{44} \rightarrow \mathrm{Ser}^{44} ; Z_{\mathrm{Mb}}=2.07\right.$; supplementary fig. S4C). By contrast, members of the terrestrial genus Episoriculus, which are nested between the semi-aquatic nectogaline lineages, exhibited secondary reductions in $Z_{\mathrm{Mb}}$ towards neutrality via different residue substitutions in each case (supplementary fig. S7A). Importantly, similar results were obtained when the above amino-acid based reconstructions were re-run on an alternative topology (supplementary fig. S3) that supported the monophyly of Episoriculus (supplementary fig. S7C).

Within the Talpidae, $Z_{\mathrm{Mb}}$ exhibited an increase from an ancestral value of 0.07 to 1.07 $\left(\mathrm{Ser}^{132} \rightarrow\right.$ Lys $^{132}$ ) in the stem Talpinae branch (fig. 5), with the probabilities of the identities of reconstructed amino acids at this node under the given tree and substitution model being 1.00 for all 153 sites (supplementary fig. S7A). With few exceptions, these values remained highly conserved in the clades containing either semi-fossorial or fossorial taxa (fig. 5 and supplementary figs. S7A, B). By contrast, $Z_{\mathrm{Mb}}$ increased to $>2$ in members of both semi-aquatic lineages - star-nosed moles and the desmans (fig. 3). For the star-nosed mole branch this entailed two charge increasing substitutions $\left(\mathrm{Asp}^{53} \rightarrow \mathrm{Gly}^{53}, \mathrm{Gln}^{128} \rightarrow \mathrm{Lys}^{128}\right.$ ) and one charge decreasing substitution (Gly ${ }^{129} \rightarrow$ Glu $^{129}$; supplementary figs. S4D and S7A). The ancestral branch of the desmans also acquired two charge increasing replacements $\left(\mathrm{Gln}^{26} \rightarrow \mathrm{Arg}^{26}, \mathrm{Asp}^{44} \rightarrow \mathrm{Ala}^{44}\right)$ together with one charge decreasing $\left(\mathrm{Asn}^{35} \rightarrow \mathrm{Asp}^{35}\right)$ replacement, with $Z_{\mathrm{Mb}}$ further being elevated in the Russian desman branch via the acquisition of an additional charge increasing 
substitution $\left(\mathrm{Gln}^{113} \rightarrow \mathrm{Lys}^{113} ; \mathrm{Z}_{\mathrm{Mb}}=3.07\right.$; figs. 3, 5). Notably, while the same residue positions are occasionally recruited in the charge altering replacements of semi-aquatic taxa, the derived residues are different in all cases (supplementary table S5).

To evaluate the above reconstructions of semi-aquatic lifestyles based on $Z_{\mathrm{Mb}}$, we coded each species as semi-aquatic or non-aquatic and estimated ancestral lifestyles using both maximum parsimony and threshold models. Although both of these latter analyses suggested that semiaquatic lifestyles evolved five times independently, this result was not strongly supported by the threshold model (supplementary fig. S9A). For example, the posterior probabilities of the most recent common ancestor of Desmana + Galemys and Chimarrogale + Nectogale being semiaquatic was only 0.85 and 0.75 , respectively, while the posterior probability for the most recent common ancestor of Nectogalini being semi-aquatic was 0.40 . To account for the alternative placement of E. fumidus, we repeated this analysis using the results of the *BEAST species tree (supplementary fig. S3). The maximum parsimony reconstruction yielded two equi-parsimonious ancestral reconstructions of semi-aquatic lifestyle in nectogaline shrews, encompassing a single origin at the base of nectogaline shrews with a secondary loss at the base of the Episoriculus clade (supplementary fig. S9B). By contrast, the threshold model only weakly supported two independent origins of a semi-aquatic lifestyle in Neomys and Nectogale + Chimarrogale.

\section{Lifestyle correlation analyses}

As a final test regarding the reliability of using $Z_{\mathrm{Mb}}$ to predict ancient semi-aquatic lifestyles, we first assigned species as semi-aquatic or non-aquatic (see fig. 2 and supplementary table S1 for lifestyle assignments), and used a threshBayes analysis to estimate covariances between $Z_{\mathrm{Mb}}$ and a semi-aquatic lifestyle. This analysis revealed a strong correlation between $Z_{\mathrm{Mb}}$ and aquatic adaptation (correlation coefficient $\mathrm{r}=0.78,95 \%$ highest posterior density $[\mathrm{HPD}]=0.48-0.93$; supplementary fig. S10A). Conversely, threshBayes analyses did not support a correlation between $Z_{\mathrm{Mb}}$ and adaptations for digging, or between $Z_{\mathrm{Mb}}$ and a fully fossorial habit, when terrestrial and semi-aquatic eulipotyphlan species were included (supplementary figs. S10B, C). When applying threshBayes to subsets of habits that included only terrestrial and fully fossorial species, or terrestrial and burrowing species, weak correlations between $Z_{\mathrm{Mb}}$ and fossoriality $(\mathrm{r}=$ $0.55)$ and digging habits $(\mathrm{r}=0.32)$ were revealed, but not significantly supported (i.e., 95\%HPD overlaps with 0 ; supplementary figs. S10D, E). Importantly, $Z_{\mathrm{Mb}}$ comparisons between semi- 
aquatic and terrestrial, and between semi-aquatic and fossorial habits (supplementary figs. S10F, G) were both significant.

\section{Discussion}

The phylogenetic estimates constructed from our comprehensive tree-of-life gene set provide a robust framework to interpret ecomorphological evolution within Eulipotyphla. The close correspondence of our concatenation and coalescent phylogenetic topologies not only support key findings of previous studies - i.e., the monophyly of shrew moles, the non-monophyly of nectogaline water shrews (Whidden 2000; He, et al. 2010; He, et al. 2017) — but finally puts to rest the long hypothesized monophyletic origin of the fully fossorial tribes Talpini and Scalopini, which have been routinely grouped together based on morphological data (see, e.g., Whidden 2000; Piras, et al. 2012; Schwermann and Thompson 2015; Hooker 2016; Sansalone, et al. 2019). Results of the present study thus provide compelling evidence that the extreme anatomical specializations for subterranean life evolved convergently in these two tribes. The molecular phylogenetic position of the amphibious desmans and semi-aquatic/fossorial star-nosed mole are also finally resolved (fig. 2), with the latter placed sister to Talpini, a relationship not supported by morphological-based hypotheses (see, e.g., Whidden 2000; Motokawa 2004; SanchezVillagra, et al. 2006).

Previous studies have failed to reach consensus on the lifestyle evolution of Eulipotyphla. Here we show that ancestral sequence reconstruction of myoglobin primary structure and $Z_{\mathrm{Mb}}$ modelling, with their well established mechanistic and biophysical underpinnings, outperform discrete, two character-state lifestyle reconstructions based on maximum parsimony and threshold approaches. These attributes, together with demonstrated links between $Z_{\mathrm{Mb}}$ and maximal Mb concentration, and hence muscle oxygen storage capacity (Mirceta, et al. 2013; Berenbrink 2020), provide strong support that this quantitative metric is well suited to resolve long-standing questions regarding lifestyle evolution within Eulipotyphla. For example, despite being less specialized for aquatic life than marine mammals, the strong positive correlation between $Z_{\mathrm{Mb}}$ and semi-aquatic specialization indicates that $Z_{\mathrm{Mb}}$ is a powerful marker to identify secondary aquatic transitions in even the world's smallest mammalian divers. Although a strong correlation was not recovered between $Z_{\mathrm{Mb}}$ and strictly fossorial habitation, $Z_{\mathrm{Mb}}$ is highly conserved in fossorial Scalopini and Talpini, with 23 of 25 species exhibiting a value of 1.07. 
413 This conservation is presumably driven by selective pressures to maintain moderately elevated tissue myoglobin levels to help foster burst burrowing activities in their hypoxic underground environment. By contrast, the $Z_{\mathrm{Mb}}$ of terrestrial species in our dataset were consistently close to neutrality, though many lineages exhibited clear signals of $Z_{\mathrm{Mb}}$ fluctuation over time

417 (supplementary figs. S7A, B, C). For example, the shrew gymnure (Neotetracus sinensis) branch evolved nine charge altering residue substitutions since its split from European hedgehogs (Erinaceus europaeus), including the charge inversion $\mathrm{Asp}^{126} \rightarrow \mathrm{Lys}^{126}$ that increases $Z_{\mathrm{Mb}}$ by +2 .

420 Similarly, the Taiwanese brown-toothed shrew branch (Episoriculus fumidus) has fourteen 421 charge altering residue substitutions, including one negative-to-positive $\left(\mathrm{Glu}^{109} \rightarrow\right.$ Lys $\left.^{109}\right)$ and two 422 positive-to-negative charge inversions ( Lys $^{102} \rightarrow \mathrm{Glu}^{102}$ and $\mathrm{Lys}^{132} \rightarrow \mathrm{Glu}^{132}$ ). These observations 423 are consistent with a stochastic evolutionary process operating under purifying selection. Charge 424 fluctuation is also apparent on the Hispaniolan solenodon (Solenodon paradoxus) branch, with 425 the moderately elevated $Z_{\mathrm{Mb}}$ value (1.85) in line with their terrestrial/burrowing lifestyle (Nowak, 426 1999). Myoglobin sequence data from additional extant/extinct members of this family (e.g., 427 Cuban solenodons) together with that from the recently extinct terrestrial/fossorial Antillean 428 family Nesophontidae (MacPhee, et al. 1999) — which is placed sister to solenodons (Brace et al. 429 2016) - may help resolve the life history evolution of this poorly understood insectivore clade.

430 Similar, albeit less pronounced charge fluctuations are evident in several fossorial mole branches 431 (as well as the stem Condylura and desman branches), suggesting that the single fossorial $Z_{\mathrm{Mb}}$ 432 outlier (hairy-tailed mole, Parascalops breweri; $Z_{\mathrm{Mb}}=2.07$ ) may also represent stochastic variation that has not yet been selected against and that presumably will return to an ecologically normalized value over evolutionary time.

435 Our results indicate that charge increases in $Z_{\mathrm{Mb}}$ to $>2$ is essential for members of eulipotyphlan mammals to successfully exploit a semi-aquatic lifestyle. Because $Z_{\mathrm{Mb}}$ of the most recent common ancestor of Talpinae was confidently estimated to be below this value (fig. 5),

438 our results do not support the "aquatic mole" hypothesis which posits that a semi-aquatic stage predated the invasion of fossorial habits in talpid moles (Campbell 1939; Whidden 1999). This conclusion is supported by the results of our lifestyle reconstructions, which uniformly revealed

441 that stem Talpinae had a low probability of semi-aquatic habits. These findings are instead 442 consistent with the interpretation that fossorial forms evolved directly from terrestrial/semi443 fossorial ancestors, without passing through a semi-aquatic phase (Reed 1951; Hickman 1984; 
444 Grand, et al. 1998). The interpretation of a semi-fossorial ancestry for early non-uropsiline

445 (Talpinae) moles is further supported by our finding of a presumed inactivation/deletion of IRBP on this branch (supplementary Table S2). This eye-specific locus has been shown to be inactivated/lost in numerous mammalian lineages that inhabit subterranean and other dim-light niches (Emerling and Springer 2014), and also appears to be non-functional in solendons and the Chinese mole shrew (this study). The $Z_{\mathrm{Mb}}$ charge increase (to 2.07) in the stem desman branch, paired with a lack of pronounced forelimb specializations for digging (He, et al. 2017) and a basal placement among non-scalopine Talpinae (fig. 2), also chimes with Reed's (1951) suggestion that the semi-aquatic lifestyle of this clade was secondary to a semi-fossorial phase. By contrast, semi-aquatic/fossorial star-nosed mole exhibits prominent morphological adaptations for burrowing and is placed sister to fossorial Talpini, consistent with Grand et al.'s (1998) hypothesis that this lineage passed through a specialized fossorial stage prior to invasion of the semi-aquatic niche. However, a semi-fossorial — as opposed to a fully fossorial — ancestry

457 for Condylura (Sansalone, et al. 2016) cannot be excluded based on the pattern of $Z_{\mathrm{Mb}}$ evolution on this branch.

The charge elevation to 1.07 in stem Nectogalini shrews is enticing, as it temporally corresponds to the early Miocene fossil Asoriculus, which was theorized to have inhabited wet environments though was unlikely to have been an efficient semi-aquatic predator (Rofes and

462 Cuenca-Bescos 2006). The single charge increasing substitution that evolved at this stage

$463\left(\mathrm{Asp}^{27} \rightarrow \mathrm{Ala}^{27}\right)$ is retained in the three semi-aquatic nectogaline genera and presumably 464 facilitated the adaptation of early Neomys and Chimarrogale + Nectogale for aquatic food resources starting some 15 and 10 million years ago, respectively. The presence of separate charge increasing replacements within the evolutionary branches of each of the three Episoriculus species also opens the possibility of additional semi-aquatic 'experiments' in this genus. Regardless, independent reductions in $Z_{\mathrm{Mb}}$ to neutrality or below for each of the latter extant species, which today inhabit damp areas in vegetated environments (Nowak 1999), is 471 foraging habit.

472 Our results provide strong evidence that the exploitation of semi-aquatic habits by extant 473 shrews and talpids occurred at least five times, and was accompanied by convergently evolved 474 charge-increasing substitutions at different surface sites on the myoglobin protein in each case 
475 (figs. 3 and 5 and supplementary figs. S4, S7). This finding provides additional support for our contention that adaptive increases in $Z_{\mathrm{Mb}}$ underlie the invasion of (semi-)aquatic niches by mammals, presumably by allowing for higher skeletal muscle myoglobin concentration (Mirceta, et al. 2013; Berenbrink 2020). This elevated $Z_{\mathrm{Mb}}$ presumably underlies the elevated $\mathrm{O}_{2}$ reserves in muscle of star-nosed moles and American water shrews compared to non-aquatic relatives (McIntyre, et al. 2002; Gusztak 2008), and likely contributes to the extended dive times and remarkable underwater foraging efficiency of these species (Catania, et al. 2008). The increase in $Z_{\mathrm{Mb}}$ must be particularly important for semi-aquatic soricine shrews due to allometic considerations that have resulted in extremely high muscle mitochondrial contents (which may comprise up to $45 \%$ of the cell volume; Weibel 1985) and mass-specific tissue $\mathrm{O}_{2}$ requirements that may be $>100$-fold higher than those of large-bodied marine mammals (Butler 1998; Gusztak, et al. 2005). It is notable that the highest $Z_{\mathrm{Mb}}$ values were found for Russian desmans (3.07) and European water shrews (3.07), consistent with the exceptional diving abilities of these species (Vogel 1998; Ivlev 2010), and it is predicted that these two species will also possess the highest muscle myoglobin concentrations among Eulipotyphla.

Adaptive evolution of similar phenotypic and physiological features occurring in distantly related lineages are not uncommon in mammals (Madsen, et al. 2001). For example, adaptive radiations in Afrotheria and Laurasiatheria resulted in striking morphological convergence of species occupying semi-aquatic (otter shrews vs. desmans) and subterranean (golden moles vs. true moles) habitats (Madsen, et al. 2001; Springer, et al. 2004). A unifying pattern underlying these and most other large-scale mammalian radiations over the past 200 million years is that they all involved ecological and locomotory diversification from ancestral lineages of small insectivores (Grossnickle, et al. 2019). The extensive radiation of small terrestrial Eulipotyphla into different adaptive zones, including four independent origins of venom systems in shrews and solenodons (Casewell, et al. 2019) and multiple independent invasions of shrews and moles to semi-aquatic, semi-fossorial, and subterranean environments that occurred on shallow timescales of only a few million years, further demonstrates the high intrinsic evolutionary potential of this Bauplan. Morphological, physiological, and even behavioral convergence have previously been identified within semi-aquatic eulipotyphlan species. For example, D. moschatus, C. cristata, and $S$. palustris can all detect prey scent while under water via the rapid exhalation and inhalation of air bubbles (Catania 2006; Catania, et al., 2008; Ivlev, et al. 2013), with the latter 
two species also being characterized by an elevated proton-buffering capacity in muscle (McIntyre, et al. 2002; Gusztak 2008). The results presented here add to this list of convergences, and indicate that semi-aquatic eulipotyphlans have evolved similar $Z_{\mathrm{Mb}}$ (and presumably elevated myoglobin concentration) phenotypes via the same selection pressure acting on different sites of the protein and by dissimilar combinations of amino acid substitutions (i.e., differential gains and losses of cationic and anionic residues, respectively). In other words, molecular adaptation of myoglobin towards life in a semi-aquatic environment is predictable at the protein level but underpinned by unpredictable genotypic evolution. As such, the phylogenomic analysis of myoglobin loci from tissue samples is not only able to provide insights into the lifestyles of rare and recently extinct mammalian species (e.g., museum specimens and subfossil material from the obscure Caribbean nesophontids), but also offers a useful tool to infer past semi-aquatic transitions based on myoglobin primary structure alone.

\section{Materials and Methods}

Eulipotyphlan taxon sampling and tree-of-life sequence data collection

Our taxon sampling of eulipotyphlan mammals included 44 talpids, 11 shrews, five erinaceids, and one solenodon (61 specimens encompassing 60 species). Note that this sampling incorporates talpid specimens from five putative 'cryptic lineages' (denoted by 'sp.', 'sp. 1', or 'sp. 2' in the figures and tables); for the purpose of this study, each of these genetically distinct lineages are considered independent species. The tissue samples were from various resources (supplementary table $\mathrm{S} 1$ ), with most tissue samples provided by co-authors from China, Japan, Canada, and the USA. Voucher specimens collected by co-authors were deposited in the Kunming Institute of Zoology (KIZ, China), the National Museum of Nature and Science (NMNS, Japan) or kept in personal collections (A.S. and S.I.K.). Additional tissue samples were obtained with permission from the National Museum of Natural History (USNM, USA), the Burke Museum of Natural History and Culture (NWBM, USA), the Field Museum of Natural History (FMNH, USA), and the New Mexico Museum of Natural History (NMMNH, USA). For each specimen, we used a capture hybridization approach (Mason, et al. 2011; Horn 2012) to enrich segments of 25 mammalian tree-of-life genes (Meredith, et al. 2011) for phylogenetic analyses. We first downloaded tree-of-life sequences from three eulipotyphlan whole genome sequences available in GenBank (Erinaceus europaeus, Sorex araneus, 
Condylura cristata), together with 60 bp of 5'- and 3'- flanking sequence for each target. We then aligned each gene segment using MAFFT (Katoh and Standley 2013). The resulting alignments were used to design 120 mer RNA probes (baits) that overlapped by 90 bp (4x tiling), and collapsed any replicates with up to six mismatches (95\% similarity) for each segment. For example, if the 120-bp gene fragments from all species were 95\% similar with each other, only one probe was designed for this region, otherwise two or more probes were designed to cover the heterogeneity. The probes were synthesized by Arbor Biosciences (Ann Arbor, MI, USA). As a first step in DNA library construction we extracted total DNA from each specimen using a Qiagen DNeasy Blood \& Tissue Kit (Qiagen, Canada). The quality and quantity of each DNA sample was measured using a Nanodrop 2000. We then sheared the total DNA into smaller fragments using NEBNext dsDNA Fragmentase (New England Biolabs, Canada), and used this as template to construct DNA libraries using a NEBNext Fast DNA Library Prep Set for Ion Torrent kit (New England Biolabs, Canada). Each sample library contained a unique barcode adapter (NEXTflex DNA Barcodes for Ion Torrent, BIOO Scientific, USA). We selected libraries within the size range of $450-500$ bp using a $\%$ E-gel on an E-Gel Electrophoresis System (Invitrogen, Canada), and re-amplified the size-selected libraries using a NEBNext HighFidelity 2X PCR Master Mix (New England Biolabs, Canada). Finally, we purified the libraries using Serapure magnetic beads, and measured DNA concentrations using a Qubit 2 Fluorometer (Thermo Fisher Scientific, Canada).

We pooled up to four DNA libraries of similar quality and concentrations before hybridization to avoid biased target captures (e.g., baits being used up by one sample). Approximately $500 \mathrm{ng}$ (100 ng to $1000 \mathrm{ng}$ ) pooled DNA library was used for each hybridization. We conducted in-solution hybridization using a myBaits ${ }^{\circledR}$ custom target capture kit (Arbor Biosciences, Ann Arbor, MI, USA) following the MYbaits user manual v3.0. The enriched libraries were re-amplified and purified as above. We thereafter measured the DNA concentration using a Qubit flourometer and pooled the enriched libraries for sequencing. The libraries were sequenced using either v318 chips on an Ion Torrent Personal Genome Machine (PGM) or an Ion PI Chip v3 via an Ion Proton Machine.

\section{Sequence assembly}


Ion Torrent sequencing technology is characterized by higher error rates than Illumina (Jünemann, et al. 2013), and Ion Torrent platforms produce single-end (rather than pair-end) reads. We therefore conducted comprehensive data cleaning and reconciliation procedures, and selected software which could handle single-end sequencing data. The raw data were automatically demultiplexed, trimmed, and converted to FASTQ format on the Torrent Suite v4.0.2 (Thermo Fisher Scientific, Canada) after sequencing. Briefly, we trimmed contaminant

573 (adapters and barcodes) sequences with AlienTrimmer (Criscuolo and Brisse 2013) using conservative parameters (-k 15 -m 5 -1 15 -q 0 -p 0 ). To remove poor quality data, we used the DynamicTrim function of the software SolexaQA++ v3.1 (Cox, et al. 2010) to trim sequences dynamically and crop the longest contiguous segment for each read. We set the probability value to 0.01 (i.e., one base call error every 100 nucleotides) in this analysis. We removed duplicated and near-duplicated reads for each sample as implemented in ParDRe using all default parameters (Gonzalez-Dominguez and Schmidt 2016). Finally, we conducted data correction using Karect, a multiple sequence alignment-based approach (Allam, et al. 2015), because this software handles substitution, insertion, and deletion errors. The output files of Karect were used for sequence assembly.

We de novo assembled the raw sequences for each sample using Abyss v2.0 (Simpson, et al. 2009), MRIA v4.0 (Chevreux, et al. 2004), and SPAdes v3.10 (Bankevich, et al. 2012), all of which were designed for short read sequencing data. Abyss is able to use a paired de Bruijn graph instead of a standard de Bruijn graph by specifying a k-mer size $(\mathrm{K})$ and a k-mer pair span (k). We set the $\mathrm{K}$ and $\mathrm{k}$ to 17 and 33, respectively, and set the maximum number of branches of a bubble to 5 in our analyses. MIRA is based on a Smith-Waterman algorithm. We ran MIRA using specific parameters including bases_per_hash $=31$ and minimum_read_length $=35$. The SPAdes assembler is also based on a de Bruijn graph, and we set only one k-mer value of 33 for analyses. It is known that merging different draft assemblies (i.e., reconciliation) could improve

592 the assembly quality (Zimin, et al. 2008). We therefore conducted reconciliation using Geneious

593 R11 (https://www.geneious.com). We concatenated the assembled draft contigs generated in three assemblers into a list. We removed contigs shorter than $120 \mathrm{bps}$, and used the BBMap 595 dedupe function to remove duplicate contigs. We conducted assemblies using the Geneious 596 assembler to group draft contigs with a minimum overlap identity of $96 \%$ to a new contig. 
Finally, all the new contigs and the leftover draft contigs were grouped into a contig list for subsequent analyses.

\section{Tree-of-life data analysis}

We additionally extracted myoglobin coding sequences and the 25 mammalian tree-of-life gene segments from seven publicly available eulipotyphlan genomes on GenBank using PHYLUCE (Faircloth 2015) and Geneious R11: Indochinese shrew (Crocidura indochinensis), gracile shrew-like mole (Uropsilus nivatus), Eastern mole (Scalopus aquaticus), Hispaniolan solenodon (Solenodon paradoxus), European hedgehog (Erinaceus europaeus), common shrew (Sorex araneus), and the star-nosed mole (Condylura cristata). Corresponding sequences from five outgroup taxa were also mined: guinea pig (Cavia porcellus), horse (Equus caballus), cat (Felis catus), pig (Sus scrofa) and bat (Pteropus alecto). PHYLUCE was originally developed for ultra-conserved elements (UCE). We followed the "harvesting UCE loci from genomes" protocol, but used the tree-of-life reference genes as probes instead of the original UCE probe sets. We extracted genomic regions which were at least $75 \%$ similar to the tree-of-life reference sequences. We also mapped the genomes to the tree-of-life references using Geneious with a minimum overlap identity of $75 \%$. These two packages were generally equally efficient at capturing target genes, though in a few cases only one successfully captured the target genes from the genome.

We used the above eulipotyphlan myoglobin and tree-of-life gene sequences to generate consensus sequences for the 61 specimens employed for the hybridization capture experiments (supplementary table S2). Briefly, the GenBank sequences were used as reference scaffolds to individually map the Ion Torrent generated reads of each sample using the Geneious "Map to Reference" function, and allowing for a mismatch of $35 \%$ per contig. This package conducts iterative mapping and outperforms many other algorithms by higher mapping rates and better consensus accuracy (Kearse, et al. 2012). Approximately 1-4 contigs from each sample were mapped to each gene reference. For the TTN gene segment, whose reference sequence was 4452 bp in length, as many as 10 contigs from each sample could be mapped to the reference. In addition, sequences of 19 nuclear gene segments obtained from 21 eulipotyphlan samples collected as part of previous studies (He, et al. 2014; He, et al. 2017), were also used for assemblies as above (and included in the final assemblies). Three shrew species (Episoriculus 
628

629

630

631

632

633

634

635

636

637

638

639

640

641

642

643

644

645

646

647

648

649

650

651

652

653

654

655

656

657

658

umbrinus, Episoriculus fumidus, and Sorex bedfordiae) for which we obtained myoglobin coding sequences via transcriptome sequencing (see below) were not included in our hybridization capture experiments. We thus downloaded the available tree-of-life genes from these species ( $A P O B, B R C A 1$, and $R A G 2$ ) on GenBank and included them in our analysis.

The resulting 25 tree-of-life gene segments were aligned separately using MAFFT. We then removed sequences shorter than $247 \mathrm{bp}$, and estimated gene trees using FastTree v2.1.5 to check for potential non-homologs for each gene. We also checked each alignment by eye, and removed ambiguous regions from the alignment. As segments of two genes (IRBP, PNOC) failed to hybridize to the majority of the DNA libraries, they were removed from subsequent analysis. The final 23 gene segment alignment (39,414 bp) included sequences from 76 samples (supplementary fig. S11). Because ASTRAL assumes no intra-locus recombination, we also tested recombination events using both RDP and GENECONV methods to examine each gene as implemented in RDP v5.5 (Martin, et al. 2015). When the program detected a signal of recombination, we checked UPGMA trees estimated using the two non-overlapping fragments and also examined the original alignment to see whether the signal was likely due to recombination or other evolutionary processes. We did not observe strong evidence of crossspecies recombination in gene alignments (data not shown).

We estimated evolutionary relationships using several different approaches. We first constructed a summary-coalescent tree whereby we simultaneously conducted rapid bootstrap analyses and searched for the best scoring maximum likelihood tree using RAXML v8.2 (Stamatakis 2014) for each gene alignment, allowing the program to determine the number of bootstraps (-\#autoMRE). We used Cavia porcellus as the root of the tree employing GTR $+\gamma$ during both ML searches and bootstrapping phases, and disabled the BFGS searching algorithm for optimizing branch lengths and GTR parameters (--no-bfgs). We followed Simmons and Kessenich's (2020) recommendation to remove dubiously supported clades and increase accuracy of tree estimation. We used RAxML to estimate SH-like aLRT support values for the bestscoring gene trees (-f j; supplementary table S7). Then we collapsed branches whose SH-like aLRT support values equal zero using Newick utilities and Tree Graph 2 (Junier and Zdobnov 2010; Stöver and Müller 2010). Then we used the collapsed trees to estimate the coalescent species tree using ASTRAL III v5.15.0 (Zhang, et al. 2018). Because gene-wise bootstrap could provide more conservative support than site-wise bootstrap analyses (Simmons, et al. 2019), we 
659

660

661

662

663

664

665

666

667

668

669

670

671

672

673

674

675

676

677

678

679

680

681

682

683

684

685

686

687

688

689

conducted gene-wise bootstrapping (--gene-only) instead of the typical site-wise bootstrapping. We also allowed the program to explore a larger search space by adding extra bipartitions to the search space (--extraLevel 2).

In addition to the summary coalescent analyses, we also constructed a concatenation-based tree using the same dataset. We partitioned the alignment by gene, searched for the best-scoring tree and conducted rapid bootstrapping under the GTR $+\gamma$ model using RAxML as described above. We used seven calibrations (supplementary text S1) and BEAST v2.5 (Bouckaert, et al. 2014) to estimate divergence times for all 76 samples as well as for the 60 species (55 eulipotyphlans and 5 outgroup species) for which complete myoglobin coding sequences were obtained. For this analysis, we first partitioned the alignment by gene and used the bModelTest package of BEAST2 to estimate the most appropriate substitution model for each gene (Bouckaert and Drummond 2017). We used a relaxed clock model with lognormal distribution for estimating the branch lengths, a birth-death model for the prior of the tree, and ran the analysis for 100 million generations. We used Cavia porcellus as the outgroup to Laurasiatheria, and also fixed the relationships of the other four outgroup species, because a biased sampling toward the ingroup (i.e., Eulipotyphla) may lead to an inaccurate estimation of outgroup relationships (Springer, et al. 2018). Secondly, we used the BModelAnalyzer package of BEAST2 to determine the best models for each gene based on the results of the bModelTest (supplementary table S6; Barido-Sottani, et al. 2018). We fixed the models of evolution based on the results of BModelAnalyzer and re-ran BEAST using the same parameters described above.

Finally, we employed a multispecies coalescent model (*BEAST; Heled and Drummond 2010) as implemented in BEAST v2.5. We grouped the samples by species. We used linear and constant root as the prior for the population model. Substitution model, tree model, and calibrations were set as above. All gene trees and species trees are given in supplementary table S8.

To examine whether alternative evolutionary hypotheses of life histories (e.g., a single origination of fully fossorial lifestyle within Talpidae) could be statistically rejected, we performed Shimodaira-Hasegawa (SH) tests. For these analyses, we constrained the monophyletic relationships of: i) fully fossorial Talpini and Scalopini moles, ii) semi-aquatic desmans and the star-nosed mole, and iii) semi-aquatic nectogaline shrew genera (Chimarrogale, Nectogale, and Neomys), one at a time and estimated the maximum likelihood concatenation 
690

691

692

693

694

695

696

697

698

699

700

701

702

703

704

705

706

707

708

709

710

711

712

713

714

715

716

717

718

719

720

trees using RAxML as described above (supplementary table S3). Then we computed the log likelihood between the best scoring maximum likelihood tree and the constrained alternative phylogenies as implemented in RAxML (-f H).

\section{Myoglobin collection and analysis}

We used four strategies to obtain myoglobin coding sequences (supplementary table S1). We first extracted available eulipotyphlan myoglobin mRNA and gene sequences from GenBank. The three coding exons were individually used both as templates for capture hybridization probe design (see above) and to map the hybridization contigs/generate consensus sequences for each exon. The 5'- and 3'- ends of introns were confirmed based on the GT-AG splice site rule. Of the 61 samples that we used for hybridization capture, complete coding sequence was obtained for 27 samples, partial myoglobin sequences were obtained for 30 samples, and no sequence obtained for 4 samples (supplementary table S1).

To cross-validate the results of our hybridization experiments, fill sequencing gaps, and extend our taxon sampling, we also PCR amplified and Sanger sequenced whole myoglobin exons from existing DNA samples and/or employed transcriptome sequencing on additional eulipotyphlan specimens. For the latter, we collected heart and lung samples from five shrews, one shrew-like mole, and one gymnure (supplementary table S1). Tissues were preserved in RNAlater (Qiagen, China), and stored at -80C. Total RNA was extracted using a RNeasy Mini kit (Qiagen, China), and mRNA subsequently enriched using immobilized oligo(dT). mRNA was sheared and reverse transcribed to DNA. The cDNA libraries were purified and re-amplified using PCR for de novo sequencing using a HiSeq $X^{\text {TM }}$ Ten Sequencing System. Approximately 6 $\mathrm{Gb}$ data were obtained for each sample. Experiments and sequencing were conducted by BioMarker Co. (Beijing, China). We used FastQC v0.11.5 (Andrews 2010) to access sequence quality, and trimmed adapter sequences using Trimmomatic v0.39 (Bolger, et al. 2014). We conducted de no assembly using Trinity v2.4 with default parameters (Grabherr, et al. 2011). Finally, primers for PCR were designed for conserved exon flanking regions from available eulipotyphlan genomes, hybridization capture, and mRNA sequences, and were used for both PCR and Sanger sequencing (supplementary table S9). These procedures resulted in complete coding sequences being obtained for 55 eulipotyphlan species (supplementary table S4). We estimated the myoglobin gene trees utilizing both nucleotide and amino acid sequences as 
implemented in RAxML. We used Dayhoff $+\gamma$ model (Dayhoff, et al. 1978) for the amino acid gene tree estimation using the same settings described above.

\section{Ancestral sequence reconstruction and homology modelling}

We estimated ancestral myoglobin sequences for each node of a 60 species phylogeny that utilized both DNA sequences and amino acids. Prior to analysis, the start (methionine) and stop codons were removed from the alignment. As in our previous study (Mirceta, et al. 2013), we performed maximum likelihood ancestral amino acid sequence reconstruction as implemented in MEGA (Kumar, et al. 2018) using the Dayhoff $+\gamma$ model that was obtained as the best-fitting substitution model using the model test facility in MEGA-X. Prior to conducting the codonbased analysis, we removed codons corresponding to residue position 121 (which was absent for 5 of the 60 species; supplementary table S4). We then used the PAML package CodeML (Yang 2007) as implemented in EasyCodeML (Gao, et al. 2019), and compared codon substitution models (site models) including M0, M1a, M2a, M3, M7, M8 and M8a using likelihood-ratio tests. We relied on the model with the highest likelihood (M8a). Because PAML does not take account of insertion/deletion events (indels), and instead treats gaps as missing data, the ancestral states of the gapped codon position 121 was reconstructed separately using a likelihood-based mixture model as implemented in FastML (Ashkenazy, et al. 2012).

To assess the three-dimensional location and any secondary or tertiary structural implications of amino acid replacements or insertions/deletions, we used the fully automated homology modelling facilities of the SWISS-MODEL server (Waterhouse, et al. 2018) to build protein structural models from the reconstructed ancestral primary structure of myoglobin in the last common eulipotyphlan ancestor and from the primary structure of the sequenced myoglobins of one species of each of the five semiaquatic lineages. Implications of the gapped position 121 on the tertiary structure of myoglobin in the Russian desman compared to the last eulipotyphlan ancestor were visualised in PyMol (The PyMOL Molecular Graphics System, Version 2.1.1, Schrödinger, LLC).

\section{Calculation of myoglobin net surface charge and electrophoretic mobility}

We calculated $Z_{\mathrm{Mb}}$ as the sum of the charge of all ionizable groups in myoglobin at $\mathrm{pH}=6.5$ by modelling $\mathrm{Mb}$ primary structures onto the tertiary structure and using published, conserved, 
site-specific ionization constants (McLellan 1984; Mirceta, et al. 2013). The reliability of modelled $Z_{\mathrm{Mb}}$ values was assessed by determining the electrophoretic mobility of native myoglobin bands at the same $\mathrm{pH}$ in muscle extracts of representative eulipotyphlan species and the grey seal, Halichoerus grypus, as an example of a marine mammal. Approximately $0.2 \mathrm{~g}$ of skeletal or cardiac muscle tissue from selected species, freed from any obvious fat or connective tissue remnants and rinsed with homogenisation buffer to move any remaining blood, was homogenized in 5 volumes of ice-cold 0.2 M MES buffer [2-(N-morpholino)ethanesulfonic acid] adjusted to $\mathrm{pH} 6.5$, using an Ultra-turrax T25 homogeniser for $10 \mathrm{~s}$ at first 9,500 rpm and then 3 times at 13,500 rpm, leaving samples to cool down between steps for $1 \mathrm{~min}$ on ice to avoid heat denaturation of proteins. The homogenized muscle extracts were then centrifuged at $10,500 \mathrm{~g}(20$ min at $4{ }^{\circ} \mathrm{C}$ ) and the supernatants stored at $-80^{\circ} \mathrm{C}$ until further use. Electrophoretic mobility of thawed muscle extracts was assessed in $9 \%$ polyacrylamide gels containing $0.3 \mathrm{M}$ MES buffer pH 6.5, using a Bio-Rad Mini-PROTEAN II gel system with 0.2 M MES pH 6.5 as the running buffer at $100 \mathrm{~V}$ and room temperature for a minimum of 3 hours. Native myoglobin bands were identified by their distinct red-brown colour before general protein staining with EZBlue ${ }^{\mathrm{TM}}$ (G104, Sigma-Aldrich). Electrophoretic mobility was assessed on digital gel images and expressed as distance travelled relative to the grey seal myoglobin, which was used as a standard of a marine mammal myoglobin with high net surface charge (Mirceta, et al. 2013). The correlation between measured relative electrophoretic mobility and modelled $Z_{\mathrm{Mb}}$ values was assessed using Phylogenetic Generalized Least Squares (PGLS) analysis using the CAPER package (Orme, et al. 2013) as implemented in R v3.6.1 and the tree from the BEAST analysis in Fig. 2. Because of low sample size, the parameter lambda was not estimated from the data but fixed at a value of 1.0.

\section{Lifestyle correlation analysis and ancestral lifestyle reconstruction}

We analyzed the relationship between lifestyles and $Z_{\mathrm{Mb}}$ based on a threshold model (Felsenstein 2012) using the phytools function threshBayes (Revell 2012) as implemented in R v3.6. The threshold model hypothesizes that each lifestyle is determined by an underlying, unobserved continuous trait (i.e., liability). We first categorized the 55 eulipotyphlan species for which $Z_{\mathrm{Mb}}$ was calculated as either semi-aquatic (including the semi-aquatic/fossorial star-nosed mole in this category) or non-aquatic based on the habits described in Burgin and He (2018). We 
783

784

785

786

787

788

789

790

791

792

793

794

795

796

797

798

799

800

801

802

803

804

805

806

807

808

809

810

811

812

813

ran Markov chain Monte Carlo (MCMC) for $10^{7}$ generations, sampling every 500 generations, and discarded the first $20 \%$ generations as burn-in. We plotted the posterior sample for the correlation to examine whether analyses reached a stationary state. We also estimated the correlation between $Z_{\mathrm{Mb}}$ and full fossoriality (i.e., fossorial species versus non-fossorial species), as well as that between $Z_{\mathrm{Mb}}$ and 'digging' (a category that included both fossorial and semifossorial species) habits using the same approach. Finally, we also created subsets of our dataset to enable comparisons between only two ecomorphotypes, with the following four threshBayes analyses conducted: terrestrial $Z_{\mathrm{Mb}}$ versus semi-aquatic $Z_{\mathrm{Mb}}$, terrestrial $Z_{\mathrm{Mb}}$ versus fully fossorial $Z_{\mathrm{Mb}}$, terrestrial $Z_{\mathrm{Mb}}$ versus semi-fossorial/fossorial $Z_{\mathrm{Mb}}$, fully fossorial $Z_{\mathrm{Mb}}$ versus semi-aquatic $Z_{\mathrm{Mb}}$

We estimated the ancestral lifestyle using a maximum parsimony and a threshold model based on the 76 species time-calibrated concatenated gene tree (fig. 2) and the *BEAST coalescent species tree (fig. S3). We categorized the species into non-aquatic or semi-aquatic as above. We used the $\mathrm{R}$ package castor to reconstruct ancestral lifestyles using maximum parsimony (Louca and Doebeli 2017), treating the transition cost between non-aquatic and semiaquatic equally. We then used the R package phytools to estimate ancestral states using a threshold model (Revell 2014). We ran MCMC for 1 million generations, sampling every 1000 generations, and discarded the first 20\% generations as burn-in. We performed the threshold analyses using either a Brownian motion or lambda model for estimating the liability, and compared the results based on deviance information criterion (DIC). We selected the result of the lambda model because it outperformed the Brownian motion model $(\Delta \mathrm{DIC}=99$, data not shown).

\section{Acknowledgements}

We thank curators and staff from the Smithsonian Institute, National Museum of Natural History (K.M. Helgen), Field Museum (L. Heaney), Burke Museum (B. Sharon) and New Mexico Museum of Natural History (B. Oh), National Museum of Natural Science (Y. Chen), Kunming Institute of Zoology (S. Li and X-L. Jiang) for approving our proposal for the use of tissue samples, or conducting destructive sampling on museum skin specimens. We are grateful to K. Wareing and S. Mirceta, University of Liverpool for muscle samples of the European mole and hedgehog, and for help with native PAGE of muscle extracts, respectively. We thank M. Docker, 
814 University of Manitoba, for use of the Ion torrent PGM, and Z.-L. Ding, Kunming Institute of

815 Zoology, for performing Ion Torrent Proton sequencing. We appreciate Ailaoshan Station for

816 Subtropical Forest Ecosystem Studies, Xishuangbanna Tropical Botanical Garden, Chinese

817 Academy of Sciences for allowing us to conduct fieldwork. We also thank Y-H. Sun and S-Y.

818 Liu for supporting our fieldwork in Gansu and Sichuan provinces, and Yuan and L-K. Lin for

819 assistance with fieldwork in Taiwan, and Z. Liu for providing tissue samples. We are grateful to

820 Y-P. Wang (Soochow university) for conducting sequencing. Finally, we are grateful to Umi

821 Matsushita for painting eulipotyphlan species for our figures. This work was supported by the

822 National Natural Science Foundation of China (31970389, 31301869 to K. H.), the National

823 Science Foundation (NSF DEB-1457735 to M.S.S), and by the University of Manitoba Research

824 Grants Program (41342), National Sciences and Engineering Research Council of Canada

825 Discovery (RGPIN/238838-2011; RGPIN/6562-2016) and Discovery Accelerator Supplement

826 (RGPIN/412336-2011) grants to K.L.C.

827

828

\section{Competing interests}

829 The authors declare that they have no conflict of interest.

830

831

\section{Data Availability Statement}

832 The newly obtained myoglobin sequences were deposited to GenBank under accession numbers

833 MW456061 to MW456069 and MW473727- MW473769, and sequence alignments per gene were deposited to Dryad Digital Repository at https://doi.org/10.5061/dryad.brv15dv7q.

835

836 Supplementary files

837 Fig. S1. The full RAxML species tree constructed from 71 eulipotyphlan specimens. Bootstrap 838 supports are given next to internal nodes.

Fig. S2. The full ASTRAL-III coalescent species tree constructed from 71 eulipotyphlan

841 specimens. Bootstrap supports are given next to internal nodes.

843 Fig. S3. The full *BEAST coalescent species tree constructed from 71 eulipotyphlan specimens.

844 Posterior probabilities are given next to internal nodes. 
846 Fig. S4. Location of charge-changing amino acid substitutions in the oxygen-storing protein myoglobin of four semi-aquatic species of moles and shrews in the mammalian insectivore order Eulipotyphla. Three-dimensional structures were obtained by homology modelling using the SWISS-MODEL server (Waterhouse, et al. 2018) and the amino acid sequences of (A) Sorex palustris, (B) Neomys fodiens, (C) Nectogale elegans, and (D) Condylura cristata. The threedimensional myoglobin structure of the last common ancestor of the group was also modelled based on results of an amino acid sequence reconstruction (see text for details). Ancestral (left) and derived (right) states of charge-changing amino acid replacements are circled and indicated with positional number and one-letter amino acid code. Blue and red color indicate amino acids with positively (H, His; K, Lys; R, Arg) and negatively charged amino acid side chains (D, Asp; E, Glu). White double arrows indicate salt bridges that are affected by charge-changing substitutions. Image views between panels A-D have been rotated to maximally visualise lineage-specific replacements.

Fig. S5: Structural model of myoglobin in (A) the last common ancestor of Eulipotyphla and (B) the semi-aquatic Russian desman (Desmana moschata). Structures were obtained by homology modelling using the SWISS-MODEL server (Waterhouse, et al. 2018) and the primary structures of myoglobin obtained by conceptual translation of the here determined nucleotide sequence (Russian desman) or by ancestral amino acid sequence reconstruction (see text for details). Structures were visualised in PyMOL version 2.1.1. Note that the gap at position 121 in the GHloop of the tertiary structure (circled in red) of the Russian desman in (B) appears to exert

Fig. S6. Time-calibrated tree of 55 eulipotyphlan species for which complete myoglobin coding 870 sequences were determined (left). Horizontal bars on the right indicate the calculated $Z_{\mathrm{Mb}}$ for each species, which are color coded according to species lifestyle.

872

873 Fig. S7. Maximum likelihood ancestral (A) amino acid-based and (B) codon-based sequence 874 reconstruction and net surface charge $\left(Z_{\mathrm{Mb}}\right)$ calculation of eulipotyphlan myoglobin mapped onto 875 the time calibrated phylogeny of fig. 2. Results of a separate amino-acid based reconstruction 
876 based on the *BEAST species tree reconstruction (supplementary fig. S3) is provided in (C) with

877 Episoriculus fumidus as the species with an alternative position compared to $A$ and $B$ in red font.

878 Only charge-changing substitutions are shown, with blue and red font in text boxes indicating

879 charge increasing and charge decreasing substitutions, respectively. Absolute charge-changing

880 substitutions $>1.0$ are underlined, whereas those $<0.10$ are shown in brackets. Yellow

881 highlighting indicates amino acids reconstructed with $\mathrm{p}<0.95$ for which less likely but

882 differentially charged amino acids have been reconstructed with $\mathrm{p}>0.05$ (see text for details).

883

884

885

886

887

888

889

890

891

892

893

894

895

896

897

898

899

900

901

902

903

904

905

906

Reconstructed net charges are shown at nodes, though have been omitted in some cases if they were identical to values on the preceding node, for clarity. Terminal species considered semiaquatic are indicated by blue font, with $Z_{\mathrm{Mb}}$ values $>+2.0$ also given in blue font.

Fig. S8. Comparisons of the Bayesian concatenation species tree estimated using the tree-of-life genes with myoglobin RAxML gene trees estimated using (A) nucleotide and (B) amino-acid sequences. Bootstrap supports are given next to internal nodes on the myoglobin gene trees. Only Bootstrap supports higher than 70 are shown.

Fig. S9. Ancestral reconstruction of semi-aquatic lifestyles (blue filled circles) within Eulipotyphla based on both the (A) RAxML concatenation gene tree and (B) the *BEAST species tree. For each tree, reconstructions were constructed using either a maximum parsimony (left) or a threshold (right) model. Note that Episoriculus fumidus (red font in $B$ ) was supported at different positions on the two trees.

Fig. S10. Posterior density distribution of the correlation coefficient (r) between (A) semiaquatic, (B) fully fossorial, and (C) digging (to include fossorial and semi-fossorial lifestyles) and $Z_{\mathrm{Mb}}$ as estimated using the threshBayes function. Figures D-G show the results based on the analyses of subsets of samples that included only: (D) terrestrial and semi-aquatic species, (E) terrestrial and fully fossorial species, (F) terrestrial and digging species, or (G) semi-aquatic and fully fossorial species. The solid red line indicates the grand mean, the green dashed lines indicate the mean of the $80 \%$ confidence intervals, and the grey dashed lines indicate the mean of the $95 \%$ confidence intervals. 
907 Fig. S11. A heatmap showing tree-of-life genes included for each of the 76 samples used for

908 phylogenetic analyses. The ultrametric tree is the BEAST concatenation gene tree and a blank

909 block indicates the gene is missing in the final dataset.

910

911 Table S1. Sample information of specimens used in this study.

912

913 Table S2. Hybridization capture results of tree-of-life gene segments from 61 eulipotyphlan

914 DNA libraries. Numbers in each column represent total base pairs captured; NA: no data.

915

Table S3. Result of likelihood-based Shimodaira-Hasegawa test to compare the best scoring

917 RAxML concatenated gene tree and alternative evolutionary hypotheses.

Table S4. Myoglobin amino acid alignment used for modeling myoglobin net surface charge

$920\left(Z_{\mathrm{Mb}}\right)$ and ancestral sequence reconstructions. Myoglobin helices A to $\mathrm{H}$ are highlighted in

921 yellow, with amino acid positions and helical notations indicated above and below the graphic,

922 respectively. Internal amino acid residue positions are shaded in light grey, while deleted

923 residues are indicated by a dash mark. Strongly anionic residues (D [Asp] and E [Glu]) are

924 shaded in red, with strongly (K [Lys] and $\mathrm{R}$ [Arg]) and weakly (H [His]) cationic residues

925 shaded in dark and light green, respectively.

927 Table S5. Charge increasing (blue font) and decreasing (red font) residue substitutions

928 reconstructed for semi-aquatic eulipotyphlan branches.

929

930 Table S6. Evolutionary models estimated using bModelTest in BEAST, and used for BEAST 931 and *BEAST analyses.

932

933 Table S7. RAxML best scoring gene trees used for ASTRAL-III coalescent analysis before and 934 after collapsing 0\% Shimodaira-Hasegawa (SH) scores in Newick format. 
Table S8. The best scoring concatenation species trees estimated using BEAST and RAxML, and the best species coalescence trees estimated using ASTRAL-III and *BEAST, in Newick format.

Table S9. Primers used to amplify and sequence the protein coding exons of myoglobin.

Text S1. Calibrations used for estimating divergence times in the BEAST analyses.

\section{References}

Allam A, Kalnis P, Solovyev V. 2015. Karect: accurate correction of substitution, insertion and deletion errors for next-generation sequencing data. Bioinformatics 31:3421-3428.

Andrews S. 2010. FastQC: a quality control tool for high throughput sequence data. In: Babraham Bioinformatics, Babraham Institute, Cambridge, United Kingdom.

Ashkenazy H, Penn O, Doron-Faigenboim A, Cohen O, Cannarozzi G, Zomer O, Pupko T. 2012. FastML: a web server for probabilistic reconstruction of ancestral sequences. Nucleic Acids Res. 40:W580-W584.

Bankevich A, Nurk S, Antipov D, Gurevich AA, Dvorkin M, Kulikov AS, Lesin VM, Nikolenko SI, Pham S, Prjibelski AD. 2012. SPAdes: a new genome assembly algorithm and its applications to single-cell sequencing. J Comput Biol. 19:455-477.

Barido-Sottani J, Boskova V, Plessis LD, Kuhnert D, Magnus C, Mitov V, Muller NF, PecErska J, Rasmussen DA, Zhang C, et al. 2018. Taming the BEAST-A community teaching material resource for BEAST 2. Syst Biol. 67:170-174.

Berenbrink M. 2020. The role of myoglobin in the evolution of mammalian diving capacity The August Krogh principle applied in molecular and evolutionary physiology. Comp Biochem Physiol A Mol Integr Physiol. 252:110843. 
968 Bolger AM, Lohse M, Usadel B. 2014. Trimmomatic: a flexible trimmer for Illumina sequence data. Bioinformatics 30:2114-2120.

970

971 Bosshard HR, Marti DN, Jelesarov I. 2004. Protein stabilization by salt bridges: concepts,

972 experimental approaches and clarification of some misunderstandings. J Mol Recognit. 17:1-16.

973

974 Bouckaert R, Heled J, Kühnert D, Vaughan T, Wu C-H, Xie D, Suchard MA, Rambaut A, 975 Drummond AJ. 2014. BEAST 2: a software platform for Bayesian evolutionary analysis. PLoS 976 Comput Biol. 10:e1003537.

977

978 Bouckaert RR, Drummond AJ. 2017. bModelTest: Bayesian phylogenetic site model averaging 979 and model comparison. BMC Evol Biol. 17:42.

Evol. 33:3095-3103.

Burgin C, He K. 2018. Family Soricidae (Shrews). In: Wilson DE, Mittermeier RA, editors.

Burgin CJ, Colella JP, Kahn PL, Upham NS. 2018. How many species of mammals are there? $J$

Mammal. 99:1-14.

991

992 Butler PM. 1998. Fossil history of shrews in Africa. In: Wojcik JM, Wolsan M, editors.

993 Evolution of Shrews. Bialowieza: Mammal Research Institute. p. 121-132.

994

995 Casewell NR, Petras D, Card DC, Suranse V, Mychajliw AM, Richards D, Koludarov I, 996 Albulescu LO, Slagboom J, Hempel BF, Ngum NM. 2019. Solenodon genome reveals 
997

convergent evolution of venom in eulipotyphlan mammals. Proc Nat Acad Sci U S A. 116:25745-25755.

Campbell B. 1939. The shoulder anatomy of the moles. A study in phylogeny and adaptation. Am J Anat. 64:1-39.

Catania KC. 2006. Olfaction - Underwater 'sniffing' by semi-aquatic mammals. Nature 444:1024-1025.

Catania KC, Hare JF, Campbell KL. 2008. Water shrews detect movement, shape, and smell to find prey underwater. Proc Nat Acad Sci U S A. 105:571-576.

Chevreux B, Pfisterer T, Drescher B, Driesel AJ, Muller WEG, Wetter T, Suhai S. 2004. Using the miraEST assembler for reliable and automated mRNA transcript assembly and SNP detection in sequenced ESTs. Genome Res. 14:1147-1159.

Churchfield S. 1990. The Natural History of Shrews: Cornell University Press.

Cox MP, Peterson DA, Biggs PJ. 2010. SolexaQA: At-a-glance quality assessment of Illumina second-generation sequencing data. BMC Bioinformatics 11:485.

Criscuolo A, Brisse S. 2013. AlienTrimmer: a tool to quickly and accurately trim off multiple short contaminant sequences from high-throughput sequencing reads. Genomics 102:500-506.

Dayhoff M, Schwartz R, Orcutt B. 1978. A model of evolutionary change in proteins. In. Atlas of protein sequence and structure: National Biomedical Research Foundation Silver Spring MD. p. 345-352.

Emerling CA, Springer MS. 2014. Eyes underground: regression of visual protein networks in subterranean mammals. Mol Phylogenet Evol. 78:260-270. 
1028

1029

1030

1031

1032

1033

1034

1035

1036

1037

1038

1039

1040

1041

1042

1043

1044

1045

1046

1047

1048

1049

1050

1051

1052

1053

1054

1055

1056

1057

1058

Enoki Y, Ohga Y, Ishidate H, Morimoto T. 2008. Primary structure of myoglobins from 31 species of birds. Comp Biochem Physiol B Biochem Mol Biol. 149:11-21.

Esselstyn JA, Oliveros CH, Swanson MT, Faircloth BC. 2017. Investigating difficult nodes in the placental mammal tree with expanded taxon sampling and thousands of ultraconserved elements. Genome Biol Evol. 9:2308-2321.

Faircloth BC. 2015. PHYLUCE is a software package for the analysis of conserved genomic loci. Bioinformatics 32:786-788.

Felsenstein J. 2012. A comparative method for both discrete and continuous characters using the threshold model. Am Nat. 179:145-156.

Gao F, Chen C, Arab DA, Du Z, He Y, Ho SYW. 2019. EasyCodeML: A visual tool for analysis of selection using CodeML. Ecol Evol. 9:3891-3898.

Gonzalez-Dominguez J, Schmidt B. 2016. ParDRe: faster parallel duplicated reads removal tool for sequencing studies. Bioinformatics 32:1562-1564.

Grabherr M, Haas BJ, Yassour M, Levin JZ, Thompson DA, Amit I, Adiconis X, Fan L, Raychowdhury R, Zeng QJNB. 2011. Full-length transcriptome assembly from RNA-Seq data without a reference genome. Nat Protoc. 29:644-652.

Gorman ML, Stone RD. 1990. The Natural History of Moles: Cornell University Press.

Grand T, Gould E, Montali R. 1998. Structure of the proboscis and rays of the star-nosed mole, Condylura cristata. J Mammal. 79:492-501.

Grossnickle DM, Smith SM, Wilson GP. 2019. Untangling the multiple ecological radiations of early mammals. Trends Ecol Evol. 34:936-949. 
1059 Gusztak RW. 2008. Dive performance and aquatic thermoregulation of the world's smallest

1060 mammalian diver, the American water shrew (Sorex palustris). M.Sc. thesis. University of

1061 Manitoba.

1062

1063 Gusztak RW, MacArthur RA, Campbell KL. 2005. Bioenergetics and thermal physiology of

1064 American water shrews (Sorex palustris). J Comp Physiol B. 175:87-95.

1065

1066

Hahn MW, Nakhleh L. 2016. Irrational exuberance for resolved species trees. Evolution 70:7-17.

1067 He K, Chen J-H, Gould GC, Yamaguchi N, Ai H-S, Wang Y-X, Zhang Y-P, Jiang X-L. 2012.

1068 An estimation of Erinaceidae phylogeny: A combined analysis approach. PLoS ONE 7:e39304.

1069

1070

He K, Li YJ, Brandley MC, Lin LK, Wang YX, Zhang YP, Jiang XL. 2010. A multi-locus

1071 phylogeny of Nectogalini shrews and influences of the paleoclimate on speciation and evolution.

1072 Mol Phylogenet Evol. 56:734-746.

1073

1074 He K, Shinohara A, Helgen KM, Springer MS, Jiang X-L, Campbell KL. 2017. Talpid mole

1075 phylogeny unites shrew moles and illuminates overlooked cryptic species diversity. Mol Biol

1076 Evol. 34:78-87.

1077

1078 He K, Shinohara A, Jiang X-L, Campbell KL. 2014. Multilocus phylogeny of talpine moles

1079 (Talpini, Talpidae, Eulipotyphla) and its implications for systematics. Mol Phylogenet Evol.

$108070: 513-521$.

1081

1082 Heled J, Drummond AJ. 2010. Bayesian inference of species trees from multilocus data. Mol

1083 Biol Evol. 27:570-580.

1084 Hickman GC. 1984. Swimming ability of talpid moles, with particular reference to the semi1085 aquatic Condylura cristata. Mammalia 48:505-514. 
1087

1088

1089

1090

1091

1092

1093

1094

1095

1096

1097

1098

1099

1100

1101

1102

1103

1104

1105

1106

1107

1108

1109

1110

1111

1112

1113

1114

1115

1116

Hooker JJ. 2016. Skeletal adaptations and phylogeny of the oldest mole Eotalpa (Talpidae, Lipotyphla, Mammalia) from the UK Eocene: the beginning of fossoriality in moles.

Palaeontology 59:195-216.

Horn S. 2012. Target enrichment via DNA hybridization capture. In: Shapiro B, Hofreiter M, editors. Ancient DNA: Springer. p. 177-188.

Hutterer R. 1985. Anatomical adaptations of shrews. Mamm Rev. 15:43-55.

Isogai $\mathrm{Y}$, Imamura $\mathrm{H}$, Nakae S, Sumi T, Takahashi K-i, Nakagawa T, Tsuneshige A, Shirai T. 2018. Tracing whale myoglobin evolution by resurrecting ancient proteins. Sci Rep. 8:16883.

Ivlev YF, Kuznetsov A, Rutovskaya M. 2010. Preliminary data on the swimming kinematics of the Russian desman (Desmana moschata L.). Dokl Biol Sci. 431:144-148.

Ivlev YF, Rutovskaya MV, Luchkina OS. (Ivlev2013 co-authors). 2013. The use of olfaction by the Russian desman (Desmana moschata L.) during underwater swimming. Dokl Biol Sci. 452:280-283.

Junemann S, Sedlazeck FJ, Prior K, Albersmeier A, John U, Kalinowski J, Mellmann A, Goesmann A, von Haeseler A, Stoye J, Harmsen D. 2013. Updating benchtop sequencing performance comparison. Nat Biotechnol. 31:294-296.

Junier T, Zdobnov EM. 2010. The Newick utilities: high-throughput phylogenetic tree processing in the UNIX shell. Bioinformatics 26:1669-1670.

Katoh K, Standley DM. 2013. MAFFT multiple sequence alignment software version 7: improvements in performance and usability. Mol Biol Evol. 30:772-780.

Kearse M, Sturrock S, Meintjes P. 2012. The Geneious 6.0. 3 read mapper. Auckland, New Zealand: Biomatters, Ltd. 
1117 Kumar S, Stecher G, Li M, Knyaz C, Tamura K. 2018. MEGA X: molecular evolutionary

1118 genetics analysis across computing platforms. Mol Biol Evol. 35:1547-1549.

1120 Lestyk KC, Folkow LP, Blix AS, Hammill MO, Burns JM. 2009. Development of myoglobin

1121 concentration and acid buffering capacity in harp (Pagophilus groenlandicus) and hooded

1122 (Cystophora cristata) seals from birth to maturity. J Comp Physiol B 179:985-996. mammals. Nature 409:610-614. recombination patterns in virus genomes. Virus Evol 1:vev003. Nesophontes: new radiometric dates and their interpretation. Am Mus Novit. 3261:1-19. and next-generation sequencing of mitochondrial genomes from noninvasively sampled museum specimens. Genome Res. 21:1695-1704.

1142 diving behaviour of the star-nosed mole (Condylura cristata) and comparisons with non-aquatic talpids. J Exp Biol. 205:45-54.

1145 McLellan T. 1984. Molecular charge and electrophoretic mobility in cetacean myoglobins of 1146 known sequence. Biochem Genet. 22:181-200. 
1148 Meredith RW, Janecka JE, Gatesy J, Ryder OA, Fisher CA, Teeling EC, Goodbla A, Eizirik E,

1149 Simao TL, Stadler T, et al. 2011. Impacts of the Cretaceous terrestrial revolution and KPg

1150 extinction on mammal diversification. Science 334:521-524.

1151

1152 Mirceta S, Signore AV, Burns JM, Cossins AR, Campbell KL, Berenbrink M. 2013. Evolution

1153 of mammalian diving capacity traced by myoglobin net surface charge. Science 340:1234192.

1154

1155 Mittermeier RA, Wilson DE, Rylands AB. 2018. Handbook of the Mammals of the World:

1156 Insectivores, Sloths and Colugos. Barcelona Lynx Edicions.

Motokawa M. 2004. Phylogenetic relationships within the family Talpidae (Mammalia: Insectivora). J Zool (Lond.) 263:147-157. W, Gribnau J, Baarends WM. 2016. Genomes of Ellobius species provide insight into the evolutionary dynamics of mammalian sex chromosomes. Genome Res. 26:1202-1210.

Noren SR, Williams TM. 2000. Body size and skeletal muscle myoglobin of cetaceans: 126:181-191. phylogenetics and evolution in R. R package version 5:1-36.

1174 Piras P, Sansalone G, Teresi L, Kotsakis T, Colangelo P, Loy A. 2012. Testing convergent and 1175 parallel adaptations in talpids humeral mechanical performance by means of geometric 1176 morphometrics and finite element analysis. J Morphol. 273:696-711. 
Ponganis PJ, Meir JU, Williams CL. 2011. In pursuit of Irving and Scholander: a review of oxygen store management in seals and penguins. J Exp Biol. 214:3325-3339. Nat. 45:513-671.

Revell LJ. 2014. Ancestral character estimation under the threshold model from quantitative genetics. Evolution 68:743-759.

Revell LJ. 2012. phytools: an R package for phylogenetic comparative biology (and other things). Methods Ecol Evol. 3:217-223. gibberodon (Petényi, 1864) in the Pleistocene of north Iberia. Riv Ital Paleontol Stratigr. 112:301-315. and the fossil record: a phylogenetic synthesis. Nature 246:389-395.

Samuel, P. P., Smith, L. P., Phillips, G. N., Jr., and Olson, J. S. (2015) Apoglobin stability Is the major factor governing both cell-free and in vivo expression of holomyoglobin. J. Biol. Chem. 290: $23479-23495$. of talpid moles (Mammalia) phylogenetic relationships. Cladistics 22:5-88.

\footnotetext{
insights about the fossil representatives of the genus. Palaeontol Electron. 19.3.54A
} 
1209 Sansalone G, Colangelo P, Loy A, Raia P, Wroe S, Piras P. 2019. Impact of transition to a 1210 subterranean lifestyle on morphological disparity and integration in talpid moles (Mammalia,

1211 Talpidae). BMC Evol Biol. 19:179.

1212

1213 Scholander P. 1962. Physiological adaptation to diving in animals and man. Harvey Lect. 57:931214110.

1215

1216 Schwermann AH, Thompson RS. 2015. Extraordinarily preserved talpids (Mammalia, 1217 Lipotyphla) and the evolution of fossoriality. J Vert Paleontol. 35:e934828. gene trees. Cladistics 36:322-340. wise resampling in phylogenetic coalescence analyses. Mol Phylogenet Evol. 131:80-92. assembler for short read sequence data. Genome Res. 19:1117-1123.

1227 Springer MS, Murphy WJ, Roca AL. 2018. Appropriate fossil calibrations and tree constraints uphold the Mesozoic divergence of solenodons from other extant mammals. Mol Phylogent Evol.

$1229 \quad 121: 158-165$. mammal tree. Trend Ecol Evol. 19:430-438.

1234 Stamatakis A. 2014. RAxML version 8: a tool for phylogenetic analysis and post-analysis of 1235 large phylogenies. Bioinformatics 30:1312-1313. 
1240 Vogel P, Bodmer C, Spreng M, Aeschimann J. 2007. Diving capacity and foraging behaviour of

1241 the water shrew. In: Gorman ML, Dunstone N, editors. Behaviour and Ecology of Riparian

1242 Mammals. New York: Cambridge University Press Cambridge, United Kingdom. p. 31-48.

1243

1244 Waterhouse A, Bertoni M, Bienert S, Studer G, Tauriello G, Gumienny R, Heer FT, de Beer

1245 TAP, Rempfer C, Bordoli L, Lepore R, Schwede T. 2018. SWISS-MODEL: homology

1246 modelling of protein structures and complexes. Nucleic Acids Res. 46:W296-W303.

1247

1248 Whidden HP. 1999. The evolution of locomotor specializations in moles. Am Zool. 39: 135A.

1250 Whidden HP. 2000. Comparative myology of moles and the phylogeny of the Talpidae

1251 (Mammalia, Lipotyphla). Am Mus Novit. 3294:1-53.

1252

1253 Woodman N, Gaffney SA. 2014. Can they dig it? Functional morphology and semifossoriality 1254 among small-eared shrews, genus Cryptotis (Mammalia, Soricidae). J Morphol. 275:745-759.

1256 Yang Z. 2007. PAML 4: phylogenetic analysis by maximum likelihood. Mol Biol Evol. 24:158612571591.

1259 Zhang C, Rabiee M, Sayyari E, Mirarab S. 2018. ASTRAL-III: polynomial time species tree 1260 reconstruction from partially resolved gene trees. BMC Bioinformatics 19:153.

1262 Zimin AV, Smith DR, Sutton G, Yorke JA. 2008. Assembly reconciliation. Bioinformatics $1263 \quad 24: 42-45$. 